Portland State University

PDXScholar

Electrical and Computer Engineering Faculty

Publications and Presentations

Electrical and Computer Engineering

$5-1-1988$

\title{
Spontaneous coherent pulsations in standing-wave laser oscillators
}

Lee W. Casperson

Portland State University

Follow this and additional works at: https://pdxscholar.library.pdx.edu/ece_fac

Part of the Electrical and Computer Engineering Commons

Let us know how access to this document benefits you.

\section{Citation Details}

Lee W. Casperson, "Spontaneous coherent pulsations in standing-wave laser oscillators," J. Opt. Soc. Am. B 5, 958-969 (1988).

This Article is brought to you for free and open access. It has been accepted for inclusion in Electrical and Computer Engineering Faculty Publications and Presentations by an authorized administrator of PDXScholar. Please contact us if we can make this document more accessible: pdxscholar@pdx.edu. 


\title{
Spontaneous coherent pulsations in standing-wave laser oscillators
}

\author{
Lee W. Casperson \\ Department of Electrical Engineering, Portland State University, Portland, Oregon 97207
}

Received September 10, 1987; accepted November 17, 1987

\begin{abstract}
Spontaneous pulsation phenomena in xenon lasers are now well known, and related effects have been observed with other laser types. Most theoretical analyses of such pulsations are formulated for unidirectional ring lasers, whereas most experiments involve standing-wave resonator geometries. Described here are rigorous semiclassical models governing the spontaneous pulsation instability in standing-wave laser oscillators. The standing-wave results are more complex and closer to experimental observations than the ring results for operation in the Lambdip region near line center.
\end{abstract}

\section{INTRODUCTION}

The existence of instabilities in laser oscillators is now a well-known phenomenon. Even before the first laser was built, spontaneous pulsations had been studied theoretically ${ }^{1}$ and had actually been observed in ruby masers. ${ }^{2,3}$ Similar effects have since been investigated with many other laser types. The earlier experimental and theoretical work on this subject has been reviewed in several places, ${ }^{4-10}$ and hence a general review is not necessary here. Instead we focus on a particular system, the xenon laser, which has also served as a model for many studies of laser instabilities. It was discovered in 1969 that under a wide range of conditions the xenon laser's output takes the form of an infinite train of pulsations. ${ }^{4}$ Sometimes the pulsations are periodic, but under other conditions period doubling and chaotic behavior are observed. The first experimental results with xenon lasers were confirmed and extended in the studies by Abraham and his co-workers. ${ }^{11-19}$

The earliest efforts to explain the xenon pulsation data were largely unsuccessful. It was found that rate-equation models for inhomogeneously broadened media could account qualitatively for the observed pulsation frequencies, but these models predicted that any initial transients would rapidly decay. The experimental data, by contrast, show no evidence of damping. Furthermore, the pulsations are sometimes highly periodic, so they cannot easily be interpreted as resulting from noise-driven fluctuations in an intrinsically stable system. Instead, the simple rate-equation model must be discarded in favor of some more rigorous formalism. In 1978 it was reported that a semiclassical model of a xenon laser did possess instabilities, and the computed pulsation waveforms had several qualitative similiarities to the experimental data. ${ }^{20}$ Those first theoretical calculations were based on a standing-wave laser model. It was soon found, however, that similar instabilities occur in unidirectional ring lasers. Because the ring-laser models are much simpler to investigate, most theoretical studies since that time have been directed toward the improved understanding of instabilities in ring lasers. The previously mentioned review articles ${ }^{4-10}$ are guides to those studies. Also, Abraham and his colleagues have reported extensive experimental studies of instability phenomena in xenon ring lasers. ${ }^{16-19}$ The most general ring-laser models include detailed numerical data for a xenon-laser discharge and seem to be able to provide good agreement with experimental data. 5,19

The successes of the ring-laser models do not, however, obviate further study of standing-wave laser systems. Most practical lasers are standing-wave devices, and many aspects of standing-wave-laser behavior cannot be represented adequately by using a ring-laser model. For example, the Lamb dip in the output power for tuning close to line center is one of the most fundamental features of gas-laser performance, and unidirectional ring models cannot exhibit this effect. Hence it could hardly be expected that a ring-laser model would provide an adequate description of any instability phenomena that might be observed when a laser is tuned close to line center. Therefore there would be considerable practical value in a standing-wave laser model that would be general enough to include the most important parameters of practical spontaneously pulsing laser oscillators.

The purpose of this study is to develop a rigorous Maxwell-Schrödinger semiclassical model for a standing-wave laser. This model is more general than our previous standing-wave semiclassical model ${ }^{20}$ in that a more realistic energy-level structure is assumed. Spectral cross relaxation due to velocity-changing collisions is also included. This inclusion of cross relaxation is essential if one wishes to obtain any accuracy in the description of the Lamb dip and the instability phenomena with which it might be associated. In fact, the model described provides good agreement with both time- and frequency-domain xenon-laser pulsation data.

The basic theoretical formalism underlying this study is developed in Section 2. The model is identical to our most general ring-laser models ${ }^{5}$ except for the choice of standingwave forms rather than traveling-wave forms for the electric field and polarization. This slight change, however, leads to a substantial increase in complexity as the atomic populations now develop a rapid $z$ dependence due to longitudinal spatial hole burning. A more convenient normalized form of the model is described in Section 3, and a variety of numerical solutions for the pulsation intensities are presented in 
Section 4. The behavior of the pulsations becomes more complex as the laser is operated further above threshold, and both the individual pulses and the average intensity are examined in the vicinity of the Lamb dip. Although the solutions were obtained by using parameter values appropriate to xenon lasers, the model itself is general enough to be applicable to many other laser types. The implications of the model for the frequency characteristics of the pulsations are reported in Ref. 21.

\section{THEORY}

The starting point for this study is the same set of MaxwellSchrödinger equations that was the basis for our recent study of ring lasers. ${ }^{5}$ The density-matrix equations of this set are

$$
\begin{aligned}
\left(\frac{\partial}{\partial t}+v \frac{\partial}{\partial z}\right) & \rho_{a b}\left(v, \omega_{\alpha}, z, t\right)=-\left(i \omega_{\alpha}+\gamma\right) \rho_{a b}\left(v, \omega_{\alpha}, z, t\right) \\
& -\frac{i \mu}{\hbar} E(z, t)\left[\rho_{a a}\left(v, \omega_{\alpha}, z, t\right)-\rho_{b b}\left(v, \omega_{\alpha}, z, t\right)\right]
\end{aligned}
$$

$$
\begin{aligned}
\left(\frac{\partial}{\partial t}+v \frac{\partial}{\partial z}\right) \rho_{a a}\left(v, \omega_{\alpha}, z, t\right)=\lambda_{a}\left(v, \omega_{\alpha}, z, t\right)-\gamma_{a} \rho_{a a}\left(v, \omega_{\alpha}, z, t\right) \\
+\left[\frac{i \mu}{\hbar} E(z, t) \rho_{b a}\left(v, \omega_{\alpha}, z, t\right)+\text { c.c. }\right] \\
-\int_{-\infty}^{\infty} \Gamma_{a}\left(v^{\prime}, v\right) \rho_{a a}\left(v, \omega_{\alpha}, z, t\right) \mathrm{d} v^{\prime} \\
+\int_{-\infty}^{\infty} \Gamma_{a}\left(v, v^{\prime}\right) \rho_{a a}\left(v^{\prime}, \omega_{\alpha}, z, t\right) \mathrm{d} v^{\prime}
\end{aligned}
$$

$$
\begin{aligned}
&\left(\frac{\partial}{\partial t}+\right.\left.v \frac{\partial}{\partial z}\right) \rho_{b b}\left(v, \omega_{\alpha}, z, t\right) \\
&= \lambda_{b}\left(v, \omega_{\alpha}, z, t\right)-\gamma_{b} \rho_{b b}\left(v, \omega_{\alpha}, z, t\right)+\gamma_{a b} \rho_{a a}\left(v, \omega_{\alpha}, z, t\right) \\
&-\left[\frac{i \mu}{\hbar} E(z, t) \rho_{b a}\left(v, \omega_{\alpha}, z, t\right)+\text { c.c. }\right] \\
&-\int_{-\infty}^{\infty} \Gamma_{b}\left(v^{\prime}, v\right) \rho_{b b}\left(v, \omega_{\alpha}, z, t\right) \mathrm{d} v^{\prime} \\
&+\int_{-\infty}^{\infty} \Gamma_{b}\left(v, v^{\prime}\right) \rho_{b b}\left(v^{\prime}, \omega_{\alpha}, z, t\right) \mathrm{d} v^{\prime}, \\
& \rho_{b a}\left(v, \omega_{\alpha}, z, t\right)=\rho_{a b} *\left(v, \omega_{\alpha}, z, t\right)
\end{aligned}
$$

where the subscripts $a$ and $b$ denote the upper and lower laser levels, respectively, $\gamma_{a}$ and $\gamma_{b}$ are the total decay rates for these levels, $\gamma_{a b}$ is the rate of direct decays from level $a$ to level $b, \gamma$ is the decay rate for the off-diagonal elements, $\lambda_{a}$ and $\lambda_{b}$ are the pumping rates, $\omega_{\alpha}$ is the center frequency of the laser transition for members of an atomic or molecular class $\alpha, \mu$ is the dipole moment for the laser transition, and the notation c.c. means the complex conjugate of the preceding terms. The integrals in Eqs. (2) and (3) represent spectral cross relaxation, and in a xenon laser this cross relaxation results from velocity-changing collisions. Thus the function $\Gamma_{a}\left(v^{\prime}, v\right) \mathrm{d} v^{\prime}$ indicates the rate at which atoms in level $a$ having velocity $v$ will be bumped by means of collisions into a velocity range between $v^{\prime}$ and $v^{\prime}+\mathrm{d} v^{\prime}$.

To the density-matrix equations for the atomic or molecular populations and polarizations must be added an equation for the electric field. The wave equation for the electric field of a linearly polarized wave in a laser medium can be written as

$$
\frac{\partial^{2} E(z, t)}{\partial z^{2}}-\mu_{1} \sigma \frac{\partial E(z, t)}{\partial t}-\mu_{1} \epsilon_{1} \frac{\partial^{2} E(z, t)}{\partial t^{2}}=\mu_{1} \frac{\partial^{2} P(z, t)}{\partial t^{2}} .
$$

The permeability $\mu_{1}$ and permittivity $\epsilon_{1}$ should be understood to include all the magnetic and dielectric properties of the laser medium except for the polarization $P$, which is due to the lasing atoms or molecules. The polarization driving this equation can be related back to the off-diagonal densitymatrix elements by

$$
P(z, t)=\int_{0}^{\infty} \int_{-\infty}^{\infty} \mu \rho_{a b}\left(v, \omega_{\alpha}, z, t\right) \mathrm{d} v \mathrm{~d} \omega_{\alpha}+\text { c.c. }
$$

Equations (1)-(6) are a complete set from which the time and space dependences of the electric field and of the atomic or molecular parameters can be determined, subject to the boundary conditions at the resonator mirrors.

The solutions of Eqs. (1)-(6) that are of interest here are those that correspond to standing-wave lasers. If the losses in a standing-wave laser can be considered to be uniformly distributed, they may be absorbed into the conductivity term. The rapid time variations in Eqs. (1)-(6) can then be factored out by means of the substitutions

$$
\begin{gathered}
E(z, t)=1 / 2 \sin (k z) E^{\prime}(t) \exp (-i \omega t)+\text { c.c. } \\
\rho_{a b}\left(v, \omega_{\alpha}, z, t\right)=P^{\prime}\left(v, \omega_{\alpha}, z, t\right) \exp (-i \omega t) / 2 \mu .
\end{gathered}
$$

Thus $\omega$ would be the actual frequency of the electromagnetic wave if $E^{\prime}$ were independent of time $t$, and the $\sin (k z)$ spatial dependence means that the laser is assumed to operate in a single longitudinal mode. With the rotating-wave approximation these substitutions reduce Eqs. (1)-(4) to the new set

$$
\begin{aligned}
&\left(\frac{\partial}{\partial t}+v \frac{\partial}{\partial z}\right) P^{\prime}\left(v, \omega_{\alpha}, z, t\right)=i\left(\omega-\omega_{\alpha}\right) P^{\prime}\left(v, \omega_{\alpha}, z, t\right) \\
&-\gamma P^{\prime}\left(v, \omega_{\alpha}, z, t\right)-\frac{i \mu^{2}}{\hbar} \sin (k z) E^{\prime}(t) \\
& \times\left[\rho_{a a}\left(v, \omega_{\alpha}, z, t\right)-\rho_{b b}\left(v, \omega_{\alpha}, z, t\right)\right], \quad(9) \\
&\left(\frac{\partial}{\partial t}+v \frac{\partial}{\partial z}\right) \rho_{a a}\left(v, \omega_{\alpha}, z, t\right)= \lambda_{a}\left(v, \omega_{\alpha}, z, t\right)-\gamma_{a} \rho_{a a}\left(v, \omega_{\alpha}, z, t\right) \\
&+\frac{i}{4 \hbar} \sin (k z)\left[E^{\prime}(t) P^{\prime *}\left(v, \omega_{\alpha}, z, t\right)\right. \\
&\left.-E^{\prime *}(t) P^{\prime}\left(v, \omega_{\alpha}, z, t\right)\right] \\
&-\int_{-\infty}^{\infty} \Gamma_{a}\left(v^{\prime}, v\right) \rho_{a a}\left(v, \omega_{\alpha}, z, t\right) \mathrm{d} v^{\prime} \\
&+\int_{-\infty}^{\infty} \Gamma_{a}\left(v, v^{\prime}\right) \rho_{a a}\left(v^{\prime}, \omega_{\alpha}, z, t\right) \mathrm{d} v^{\prime}
\end{aligned}
$$




$$
\begin{aligned}
\left(\frac{\partial}{\partial t}+v \frac{\partial}{\partial z}\right) \rho_{b b}\left(v, \omega_{\alpha}, z, t\right)= & \lambda_{b}\left(v, \omega_{\alpha}, z, t\right)-\gamma_{b} \rho_{b b}\left(v, \omega_{\alpha}, z, t\right) \\
& +\gamma_{a b} \rho_{a a}\left(v, \omega_{\alpha}, z, t\right) \\
& -\frac{i}{4 \hbar} \sin (k z)\left[E^{\prime}(t) P^{\prime *}\left(v, \omega_{\alpha}, z, t\right)\right. \\
& \left.-E^{\prime *}(t) P^{\prime}\left(v, \omega_{\alpha}, z, t\right)\right] \\
& -\int_{-\infty}^{\infty} \Gamma_{b}\left(v^{\prime}, v\right) \rho_{b b}\left(v, \omega_{\alpha}, z, t\right) \mathrm{d} v^{\prime} \\
& +\int_{-\infty}^{\infty} \Gamma_{b}\left(v, v^{\prime}\right) \rho_{b b}\left(v^{\prime}, \omega_{\alpha}, z, t\right) \mathrm{d} v^{\prime} .
\end{aligned}
$$

The same substitutions reduce Eqs. (5) and (6) to the new field equation

where the field and polarization amplitudes are assumed to vary slowly enough in time that their higher derivatives can be neglected.

It is convenient to introduce the new frequency parameter $\Omega=k\left(\mu_{1} \epsilon_{1}\right)^{-1 / 2}$ so that Eq. (12) can be written as

$$
\begin{array}{r}
\sin (k z)\left[i \frac{\mathrm{d} E^{\prime}(t)}{\mathrm{d} t}+\frac{i \sigma}{2 \epsilon_{1}} E^{\prime}(t)+\frac{\omega^{2}-\Omega^{2}}{2 \omega} E^{\prime}(t)\right] \\
=-\frac{\omega}{2 \epsilon_{1}} \int_{0}^{\infty} \int_{-\infty}^{\infty} P^{\prime}\left(v, \omega_{\alpha}, z, t\right) \mathrm{d} v \mathrm{~d} \omega_{\alpha} .
\end{array}
$$

It is clear from the form of Eq. (13) that $\Omega$ can be interpreted as the nondispersed cavity frequency, i.e., the steady-state lasing frequency if the dispersion or real part of $P^{\prime}$ were equal to zero. Because the assumed lasing frequency $\omega$ is also close to the nondispersed frequency $\Omega$, the term $\left(\omega^{2}-\right.$ $\left.\Omega^{2}\right) / 2 \omega$ can be approximated by $\omega-\Omega$. Because the lasing

$$
\begin{array}{r}
\sin (k z)\left[i \mu_{1} \epsilon_{1} \omega \frac{\mathrm{d} E^{\prime}(t)}{\mathrm{d} t}+i \frac{\mu_{1} \sigma \omega}{2} E^{\prime}(t)+\left(\frac{\omega^{2} \mu_{1} \epsilon_{1}}{2}-\frac{k^{2}}{2}\right) E^{\prime}(t)\right] \\
=-\frac{\mu_{1} \omega^{2}}{2} \int_{0}^{\infty} \int_{-\infty}^{\infty} P^{\prime}\left(v, \omega_{\alpha}, z, t\right) \mathrm{d} v \mathrm{~d} \omega_{\alpha},
\end{array}
$$

frequency is also close to the transition frequency, the $\omega$ multiplying the polarization integral can be replaced by $\omega_{0}$, a characteristic line center frequency of the transition. Multiplying Eq. (13) by $\sin (k z)$ and integrating over the length $L$ of the cavity now yields

$$
\begin{aligned}
\frac{\mathrm{d} E^{\prime}(t)}{\mathrm{d} t}= & -\frac{\sigma}{2 \epsilon_{1}} E^{\prime}(t)+i(\omega-\Omega) E^{\prime}(t) \\
& +i \frac{\omega_{0}}{2 \epsilon_{1}} \int_{0}^{\infty} \int_{-\infty}^{\infty} \int_{0}^{L} \sin (k z) P^{\prime}\left(v, \omega_{\alpha}, z, t\right) \mathrm{d} z \mathrm{~d} v \mathrm{~d} \omega_{\alpha} .
\end{aligned}
$$

Equations (9)-(11) and (14) are a set of first-order differential equations governing the laser behavior. These equations are complicated by the fact that the electric field $E^{\prime}(t)$ and the polarization $P^{\prime}\left(v, \omega_{\alpha}, z, t\right)$ are complex quantities. For computational purposes we find it helpful to express these quantities in terms of their real and imaginary parts according to

$$
P^{\prime}\left(v, \omega_{\alpha}, z, t\right)=P_{r}\left(v, \omega_{\alpha}, z, t\right)+i P_{i}\left(v, \omega_{\alpha}, z, t\right)
$$

and

$$
E^{\prime}(t)=E_{r}(t)+i E_{i}(t) .
$$

With these substitutions the governing equations reduce to the real set

$$
\begin{aligned}
\left(\frac{\partial}{\partial t}+v \frac{\partial}{\partial z}\right) P_{r}\left(v, \omega_{\alpha}, z, t\right)= & -\left(\omega-\omega_{\alpha}\right) P_{i}\left(v, \omega_{\alpha}, z, t\right) \\
& -\gamma P_{r}\left(v, \omega_{\alpha}, z, t\right) \\
& +\frac{\mu^{2}}{\hbar} \sin (k z) E_{i}(t) D\left(v, \omega_{\alpha}, z, t\right),
\end{aligned}
$$

$$
\begin{aligned}
\left(\frac{\partial}{\partial t}+v \frac{\partial}{\partial z}\right) P_{i}\left(v, \omega_{\alpha}, z, t\right)= & \left(\omega-\omega_{\alpha}\right) P_{r}\left(v, \omega_{\alpha}, z, t\right) \\
& -\gamma P_{i}\left(v, \omega_{\alpha}, z, t\right) \\
& -\frac{\mu^{2}}{\hbar} \sin (k z) E_{r}(t) D\left(v, \omega_{\alpha}, z, t\right),
\end{aligned}
$$

$$
\begin{aligned}
\left(\frac{\partial}{\partial t}+v \frac{\partial}{\partial z}\right) D\left(v, \omega_{\alpha}, z, t\right) & =\lambda_{a}\left(v, \omega_{\alpha}, z, t\right)-\lambda_{b}\left(v, \omega_{\alpha}, z, t\right)-\frac{\gamma_{a}+\gamma_{a b}+\gamma_{b}}{2} D\left(v, \omega_{\alpha}, z, t\right) \\
& -\frac{\gamma_{a}+\gamma_{a b}-\gamma_{b}}{2} M\left(v, \omega_{\alpha}, z, t\right)+\frac{\sin (k z)}{\hbar}\left[E_{r}(t) P_{i}\left(v, \omega_{\alpha}, z, t\right)-E_{i}(t) P_{r}\left(v, \omega_{\alpha}, z, t\right)\right] \\
& -\frac{1}{2} \int_{-\infty}^{\infty} \Gamma_{a}\left(v^{\prime}, v\right)\left[M\left(v, \omega_{\alpha}, z, t\right)+D\left(v, \omega_{\alpha}, z, t\right)\right] \mathrm{d} v^{\prime}+\frac{1}{2} \int_{-\infty}^{\infty} \Gamma_{a}\left(v, v^{\prime}\right)\left[M\left(v^{\prime}, \omega_{\alpha}, z, t\right)+D\left(v^{\prime}, \omega_{\alpha}, z, t\right)\right] \mathrm{d} v^{\prime} \\
& +\frac{1}{2} \int_{-\infty}^{\infty} \Gamma_{b}\left(v^{\prime}, v\right)\left[M\left(v, \omega_{\alpha}, z, t\right)-D\left(v, \omega_{\alpha}, z, t\right)\right] \mathrm{d} v^{\prime}-\frac{1}{2} \int_{-\infty}^{\infty} \Gamma_{b}\left(v, v^{\prime}\right)\left[M\left(v^{\prime}, \omega_{\alpha}, z, t\right)-D\left(v^{\prime}, \omega_{\alpha}, z, t\right)\right] \mathrm{d} v^{\prime}
\end{aligned}
$$

$$
\begin{aligned}
\left(\frac{\partial}{\partial t}+v \frac{\partial}{\partial z}\right) M\left(v, \omega_{\alpha}, z, t\right)= & \lambda_{a}\left(v, \omega_{\alpha}, z, t\right)+\lambda_{b}\left(v, \omega_{\alpha}, z, t\right)-\frac{\gamma_{a}-\gamma_{a b}-\gamma_{b}}{2} D\left(v, \omega_{\alpha}, z, t\right)-\frac{\gamma_{a}-\gamma_{a b}+\gamma_{b}}{2} M\left(v, \omega_{\alpha}, z, t\right) \\
& -\frac{1}{2} \int_{-\infty}^{\infty} \Gamma_{a}\left(v^{\prime}, v\right)\left[M\left(v, \omega_{\alpha}, z, t\right)+D\left(v, \omega_{\alpha}, z, t\right)\right] \mathrm{d} v^{\prime}+\frac{1}{2} \int_{-\infty}^{\infty} \Gamma_{a}\left(v, v^{\prime}\right)\left[M\left(v^{\prime}, \omega_{\alpha}, z, t\right)+D\left(v^{\prime}, \omega_{\alpha}, z, t\right)\right] \mathrm{d} v^{\prime} \\
& -\frac{1}{2} \int_{-\infty}^{\infty} \Gamma_{b}\left(v^{\prime}, v\right)\left[M\left(v, \omega_{\alpha}, z, t\right)-D\left(v, \omega_{\alpha}, z, t\right)\right] \mathrm{d} v^{\prime}+\frac{1}{2} \int_{-\infty}^{\infty} \Gamma_{b}\left(v, v^{\prime}\right)\left[M\left(v^{\prime}, \omega_{\alpha}, z, t\right)-D\left(v^{\prime}, \omega_{\alpha}, z, t\right)\right] \mathrm{d} v^{\prime},
\end{aligned}
$$




$$
\begin{aligned}
\frac{\mathrm{d} E_{r}(t)}{\mathrm{d} t}= & \frac{E_{r}(t)}{2 t_{c}}-(\omega-\Omega) E_{i}(t) \\
& -\frac{\omega_{0}}{\epsilon_{1} L} \int_{0}^{\infty} \int_{-\infty}^{\infty} \int_{0}^{l} \sin (k z) P_{i}\left(v, \omega_{\alpha}, z, t\right) \mathrm{d} z \mathrm{~d} v \mathrm{~d} \omega_{\alpha} \\
\frac{\mathrm{d} E_{i}(t)}{\mathrm{d} t}=- & \frac{E_{i}(t)}{2 t_{c}}+(\omega-\Omega) E_{r}(t) \\
& +\frac{\omega_{0}}{\epsilon_{1} L} \int_{0}^{\infty} \int_{-\infty}^{\infty} \int_{0}^{l} \sin (k z) P_{r}\left(v, \omega_{\alpha}, z, t\right) \mathrm{d} z \mathrm{~d} v \mathrm{~d} \omega_{\alpha}
\end{aligned}
$$

where we have introduced the population difference $D\left(v, \omega_{\alpha}\right.$, $z, t)=\rho_{a a}\left(v, \omega_{\alpha}, z, t\right)-\rho_{b b}\left(v, \omega_{\alpha}, z, t\right)$, the population sum $M\left(v, \omega_{\alpha}, z, t\right)=\rho_{a a}\left(v, \omega_{\alpha}, z, t\right)+\rho_{b b}\left(v, \omega_{\alpha}, z, t\right)$, and the cavity lifetime $t_{c}=\epsilon_{1} / \sigma$. Also, the length integration extends only over the amplifier length $l$. We note that for a single-mode laser in which the amplifier is long compared with the wavelength, the position of the amplifier within the resonator is unimportant.

Next, it is necessary to make some assumptions about the form of the spectral cross-relaxation kernels. For simplicity and consistency with our ring-laser studies, it is considered that the cross-relaxation integrals apply to strong collisions in which the final velocities are distributed randomly across the Doppler profile. Thus the kernels can be written as

$$
\begin{aligned}
& \Gamma_{a}\left(v, v^{\prime}\right)=\frac{\Gamma_{a}}{u \pi^{1 / 2}} \exp \left(-v^{2} / u^{2}\right), \\
& \Gamma_{b}\left(v, v^{\prime}\right)=\frac{\Gamma_{b}}{u \pi^{1 / 2}} \exp \left(-v^{2} / u^{2}\right),
\end{aligned}
$$

where, for example, $\Gamma_{a}$ is the total rate at which atoms in level $a$ undergo strong velocity-changing collisions and $u$ is the most probable speed of the atoms. With these substitutions, Eqs. (17) and (18) for the population difference and sum become

$$
\begin{aligned}
\left(\frac{\partial}{\partial t}+v \frac{\partial}{\partial z}\right) & D\left(v, \omega_{\alpha}, z, t\right)=\lambda_{a}\left(v, \omega_{\alpha}, z, t\right) \\
& -\lambda_{b}\left(v, \omega_{\alpha}, z, t\right)-\frac{\gamma_{a}^{\prime}+\gamma_{a b}+\gamma_{b}^{\prime}}{2} D\left(v, \omega_{\alpha}, z, t\right) \\
& -\frac{\gamma_{a}{ }^{\prime}+\gamma_{a b}-\gamma_{b}^{\prime}}{2} M\left(v, \omega_{\alpha}, z, t\right) \\
& +\frac{\sin (k z)}{\hbar}\left[E_{r}(t) P_{i}\left(v, \omega_{\alpha}, z, t\right)-E_{i}(t) P_{r}\left(v, \omega_{\alpha}, z, t\right)\right] \\
& +\frac{\Gamma_{a}}{2 u \pi^{1 / 2}} \exp \left(-v^{2} / u^{2}\right) \int_{-\infty}^{\infty}\left[M\left(v^{\prime}, \omega_{\alpha}, z, t\right)\right. \\
& \left.+D\left(v^{\prime}, \omega_{\alpha}, z, t\right)\right] \mathrm{d} v^{\prime}-\frac{\Gamma_{b}}{2 u \pi^{1 / 2}} \exp \left(-v^{2} / u^{2}\right) \\
& \times \int_{-\infty}^{\infty}\left[M\left(v^{\prime}, \omega_{\alpha}, z, t\right)-D\left(v^{\prime}, \omega_{\alpha}, z, t\right)\right] \mathrm{d} v^{\prime},
\end{aligned}
$$

$$
\begin{aligned}
& \left(\frac{\partial}{\partial t}+v \frac{\partial}{\partial z}\right) M\left(v, \omega_{\alpha}, z, t\right)=\lambda_{a}\left(v, \omega_{\alpha}, z, t\right) \\
& \quad+\lambda_{b}\left(v, \omega_{\alpha}, z, t\right)-\frac{\gamma_{a}^{\prime}-\gamma_{a b}-\gamma_{b}^{\prime}}{2} D\left(v, \omega_{\alpha}, z, t\right) \\
& \quad-\frac{\gamma_{a}^{\prime}-\gamma_{a b}+\gamma_{b}^{\prime}}{2} M\left(v, \omega_{\alpha}, z, t\right) \\
& +\frac{\Gamma_{a}}{2 u \pi^{1 / 2}} \exp \left(-v^{2} / u^{2}\right) \int_{-\infty}^{\infty}\left[M\left(v^{\prime}, \omega_{\alpha}, z, t\right)+D\left(v^{\prime}, \omega_{\alpha}, z, t\right)\right] \mathrm{d} v^{\prime} \\
& +\frac{\Gamma_{b}}{2 u \pi^{1 / 2}} \exp \left(-v^{2} / u^{2}\right) \int_{-\infty}^{\infty}\left[M\left(v^{\prime}, \omega_{\alpha}, z, t\right)-D\left(v^{\prime}, \omega_{\alpha}, z, t\right)\right] \mathrm{d} v^{\prime}
\end{aligned}
$$

where the modified decay rates are defined by $\gamma_{a}{ }^{\prime}=\gamma_{a}+\Gamma_{a}$ and $\gamma_{b}{ }^{\prime}=\gamma_{b}+\Gamma_{b}$. Equations (15), (16), (19), (20), (23), and (24) are a complete set governing the time- and space-dependent field, polarization, and populations. , For numerical reasons, however, it proves helpful to introduce certain additional transformations.

One particular difficulty with the model described above is that it includes partial derivatives with respect to both the space and time variables. To proceed with the solution we could consider mathematically breaking up the length of the laser into many small but finite length segments and writing distinct equations for the polarization and population variables in each segment. Because of the high spatial frequency of the fields in most lasers, however, it is far more efficient to decompose the polarization and the population into a series of spatial harmonics of the electric field. Thus we introduce the expansions

$$
\begin{aligned}
& P_{r}\left(v, \omega_{\alpha}, z, t\right)=\sum_{j=-\infty}^{\infty} P_{r, 2 j+1}\left(v, \omega_{\alpha}, t\right) \exp [(2 j+1) i k z] \\
& P_{i}\left(v, \omega_{\alpha}, z, t\right)=\sum_{j=-\infty}^{\infty} P_{i, 2 j+1}\left(v, \omega_{\alpha}, t\right) \exp [(2 j+1) i k z] \\
& D\left(v, \omega_{\alpha}, z, t\right)=\sum_{j=-\infty}^{\infty} D_{2 j}\left(v, \omega_{\alpha}, t\right) \exp [(2 j) i k z] \\
& M\left(v, \omega_{\alpha}, z, t\right)=\sum_{j=-\infty}^{\infty} M_{2 j}\left(v, \omega_{\alpha}, t\right) \exp [(2 j) i k z]
\end{aligned}
$$

where only odd harmonics of the polarizations and even harmonics of the populations are included. It is clear physically that the polarizations must be spatially in phase with the field, i.e., odd, and the populations must be spatially out of phase, i.e., even, provided that the pump rates are also spatially even. In this analysis we make the usual assumption that the pump rates are spatially uniform, and thus no additional harmonics are required. The left-hand sides of Eqs. (25)-(28) must be real. This imposes additional constraints on the expansion coefficients, and these constraints can be written as

$$
\begin{aligned}
& P_{r, j}\left(v, \omega_{\alpha}, t\right)=P_{r,-j} *\left(v, \omega_{\alpha}, t\right), \\
& P_{i, j}\left(v, \omega_{\alpha}, t\right)=P_{i,-j}^{*}\left(v, \omega_{\alpha}, t\right),
\end{aligned}
$$




$$
\begin{aligned}
& D_{j}\left(v, \omega_{\alpha}, t\right)=D_{-j}^{*}\left(v, \omega_{\alpha}, t\right), \\
& M_{j}\left(v, \omega_{\alpha}, t\right)=M_{-j}^{*}\left(v, \omega_{\alpha}, t\right) .
\end{aligned}
$$

When Eqs. (25)-(32) are substituted into Eqs. (15), (16), (19), (20), (23), and (24), we obtain the new set

$$
\begin{aligned}
\frac{\partial P_{r, 2 j+1}\left(v, \omega_{\alpha}, t\right)}{\partial t}= & -\left(\omega-\omega_{\alpha}\right) P_{i, 2 j+1}\left(v, \omega_{\alpha}, t\right) \\
& -[(2 j+1) i k v+\gamma] P_{r, 2 j+1}\left(v, \omega_{\alpha}, t\right) \\
& -\frac{i \mu^{2}}{2 \hbar} E_{i}(t)\left[D_{2 j}\left(v, \omega_{\alpha}, t\right)-D_{2 j+2}\left(v, \omega_{\alpha}, t\right)\right],
\end{aligned}
$$

$$
\begin{aligned}
\frac{\partial P_{i, 2 j+1}\left(v, \omega_{\alpha}, t\right)}{\partial t}= & \left(\omega-\omega_{\alpha}\right) P_{r, 2 j+1}\left(v, \omega_{\alpha}, t\right) \\
& -[(2 j+1) i k v+\gamma] P_{i, 2 j+1}\left(v, \omega_{\alpha}, t\right) \\
& +\frac{i \mu^{2}}{2 \hbar} E_{r}(t)\left[D_{2 j}\left(v, \omega_{\alpha}, t\right)-D_{2 j+2}\left(v, \omega_{\alpha}, t\right)\right],
\end{aligned}
$$

out the -1 and +1 spatial harmonics of the polarizations, since the higher-order components average to zero over long distances. The advantage of this expanded form of the laser equations is that the $z$ derivatives and integrations have all been eliminated. Equations (33)-(38) are the basis for all of our standing-wave laser calculations.

\section{NORMALIZED EQUATIONS}

It is helpful to rewrite the laser equations in a normalized form. A particularly convenient normalization can be obtained from the steady-state version of these equations. The steady-state forms of Eqs. (33)-(38) are

$$
\begin{gathered}
0=-\left(\omega-\omega_{\alpha}\right) P_{i, 2 j+1}\left(v, \omega_{\alpha}\right)-[(2 j+1) i k v+\gamma] P_{r, 2 j+1}\left(v, \omega_{\alpha}\right) \\
-\frac{i \mu^{2}}{2 \hbar} E_{i}\left[D_{2 j}\left(v, \omega_{\alpha}\right)-D_{2 j+2}\left(v, \omega_{\alpha}\right)\right], \\
0=\left(\omega-\omega_{\alpha}\right) P_{r, 2 j+1}\left(v, \omega_{\alpha}\right)-[(2 j+1) i k v+\gamma] P_{i, 2 j+1}\left(v, \omega_{\alpha}\right) \\
+\frac{i \mu^{2}}{2 \hbar} E_{r}\left[D_{2 j}\left(v, \omega_{\alpha}\right)-D_{2 j+2}\left(v, \omega_{\alpha}\right)\right],
\end{gathered}
$$

$$
\begin{aligned}
& \frac{\partial D_{2 j}\left(v, \omega_{\alpha}, t\right)}{\partial t}=\left[\lambda_{a}\left(v, \omega_{\alpha}, t\right)-\lambda_{b}\left(v, \omega_{\alpha}, t\right)\right] \delta_{j 0}-\left[(2 j) i k v+h_{1}\right] D_{2 j}\left(v, \omega_{\alpha}, t\right)-h_{2} M_{2 j}\left(v, \omega_{\alpha}, t\right) \\
& \quad-\frac{i}{2 \hbar}\left\{\left[E_{r}(t) P_{i, 2 j-1}\left(v, \omega_{\alpha}, t\right)-E_{i}(t) P_{r, 2 j-1}\left(v, \omega_{\alpha}, t\right)\right]-\left[E_{r}(t) P_{i, 2 j+1}\left(v, \omega_{\alpha}, t\right)-E_{i}(t) P_{r, 2 j+1}\left(v, \omega_{\alpha}, t\right)\right]\right\} \\
& \quad+\frac{\Gamma_{a}}{2 u \pi^{1 / 2}} \exp \left(-v^{2} / u^{2}\right) \int_{-\infty}^{\infty}\left[M_{2 j}\left(v^{\prime}, \omega_{\alpha}, t\right)+D_{2 j}\left(v^{\prime}, \omega_{\alpha}, t\right)\right] \mathrm{d} v^{\prime}-\frac{\Gamma_{b}}{2 u \pi^{1 / 2}} \exp \left(-v^{2} / u^{2}\right) \int_{-\infty}^{\infty}\left[M_{2 j}\left(v^{\prime}, \omega_{\alpha}, t\right)-D_{2 j}\left(v^{\prime}, \omega_{\alpha}, t\right)\right] \mathrm{d} v^{\prime},
\end{aligned}
$$

$$
\begin{aligned}
& \frac{\partial M_{2 j}\left(v, \omega_{\alpha}, t\right)}{\partial t}=\left[\lambda_{a}\left(v, \omega_{\alpha}, t\right)+\lambda_{b}\left(v, \omega_{\alpha}, t\right)\right] \delta_{j 0}-\left[(2 j) i k v+h_{3}\right] M_{2 j}\left(v, \omega_{\alpha}, t\right)-h_{4} D_{2 j}\left(v, \omega_{\alpha}, t\right) \\
& \quad+\frac{\Gamma_{a}}{2 u \pi^{1 / 2}} \exp \left(-v^{2} / u^{2}\right) \int_{-\infty}^{\infty}\left[M_{2 j}\left(v^{\prime}, \omega_{\alpha}, t\right)+D_{2 j}\left(v^{\prime}, \omega_{\alpha}, t\right)\right] \mathrm{d} v^{\prime}+\frac{\Gamma_{b}}{2 u \pi^{1 / 2}} \exp \left(-v^{2} / u^{2}\right) \int_{-\infty}^{\infty}\left[M_{2 j}\left(v^{\prime}, \omega_{\alpha}, t\right)-D_{2 j}\left(v^{\prime}, \omega_{\alpha}, t\right)\right] \mathrm{d} v^{\prime},
\end{aligned}
$$

$$
\begin{aligned}
\frac{\mathrm{d} E_{r}(t)}{\mathrm{d} t}= & -\frac{E_{r}(t)}{2 t_{c}}-(\omega-\Omega) E_{i}(t) \\
& -\frac{\omega_{0} l}{\epsilon_{1} L} \int_{0}^{\infty} \int_{-\infty}^{\infty} \frac{P_{i,-1}\left(v, \omega_{\alpha}, t\right)-P_{i, 1}\left(v, \omega_{\alpha}, t\right)}{2 i} \mathrm{~d} v \mathrm{~d} \omega_{\alpha}
\end{aligned}
$$

$$
\begin{aligned}
\frac{\mathrm{d} E_{i}(t)}{\mathrm{d} t}= & -\frac{E_{i}(t)}{2 t_{c}}+(\omega-\Omega) E_{r}(t) \\
& +\frac{\omega_{0} l}{\epsilon_{1} L} \int_{0}^{\infty} \int_{-\infty}^{\infty} \frac{P_{r,-1}\left(v, \omega_{\alpha}, t\right)-P_{r, 1}\left(v, \omega_{\alpha}, t\right)}{2 i} \mathrm{~d} v \mathrm{~d} \omega_{\alpha},
\end{aligned}
$$

where the decay rates have been replaced by the new parameters

$$
\begin{aligned}
& h_{1}=\left(\gamma_{a}{ }^{\prime}+\gamma_{a b}+\gamma_{b}{ }^{\prime}\right) / 2, \\
& h_{2}=\left(\gamma_{a}{ }^{\prime}+\gamma_{a b}-\gamma_{b}{ }^{\prime}\right) / 2, \\
& h_{3} \equiv\left(\gamma_{a}{ }^{\prime}-\gamma_{a b}+\gamma_{b}{ }^{\prime}\right) / 2, \\
& h_{4}=\left(\gamma_{a}{ }^{\prime}-\gamma_{a b}-\gamma_{b}{ }^{\prime}\right) / 2 .
\end{aligned}
$$

Also, the $z$ integrations in the field equations have selected

$$
\begin{aligned}
0= & {\left[\lambda_{a}\left(v, \omega_{\alpha}\right)-\lambda_{b}\left(v, \omega_{\alpha}\right)\right] \delta_{j 0}-\left[(2 j) i k v+h_{1}\right] D_{2 j}\left(v, \omega_{\alpha}\right) } \\
& -h_{2} M_{2 j}\left(v, \omega_{\alpha}\right)-\frac{i}{2 \hbar}\left\{\left[E_{r} P_{i, 2 j-1}\left(v, \omega_{\alpha}\right)\right.\right. \\
& \left.\left.-E_{i} P_{r, 2 j-1}\left(v, \omega_{\alpha}\right)\right]-\left[E_{r} P_{i, 2 j+1}\left(v, \omega_{\alpha}\right)-E_{i} P_{r, 2 j+1}\left(v, \omega_{\alpha}\right)\right]\right\} \\
& +\frac{\Gamma_{a}}{2 u \pi^{1 / 2}} \exp \left(-v^{2} / u^{2}\right) \int_{-\infty}^{\infty}\left[M_{2 j}\left(v^{\prime}, \omega_{\alpha}\right)+D_{2 j}\left(v^{\prime}, \omega_{\alpha}\right)\right] \mathrm{d} v^{\prime}, \\
& -\frac{\Gamma_{b}}{2 u \pi^{1 / 2}} \exp \left(-v^{2} / u^{2}\right) \int_{-\infty}^{\infty}\left[M_{2 j}\left(v^{\prime}, \omega_{\alpha}\right)-D_{2 j}\left(v^{\prime}, \omega_{\alpha}\right)\right] \mathrm{d} v^{\prime},
\end{aligned}
$$

$$
\begin{aligned}
0= & {\left[\lambda_{a}\left(v, \omega_{\alpha}\right)+\lambda_{b}\left(v, \omega_{\alpha}\right)\right] \delta_{j 0}-\left[(2 j) i k v+h_{3}\right] M_{2 j}\left(v, \omega_{\alpha}\right) } \\
& -h_{4} D_{2 j}\left(v, \omega_{\alpha}\right) \\
& +\frac{\Gamma_{a}}{2 u \pi^{1 / 2}} \exp \left(-v^{2} / u^{2}\right) \int_{-\infty}^{\infty}\left[M_{2 j}\left(v^{\prime}, \omega_{\alpha}\right)+D_{2 j}\left(v^{\prime}, \omega_{\alpha}\right)\right] \mathrm{d} v^{\prime}
\end{aligned}
$$$$
+\frac{\Gamma_{b}}{2 u \pi^{1 / 2}} \exp \left(-v^{2} / u^{2}\right) \int_{-\infty}^{\infty}\left[M_{2 j}\left(v^{\prime}, \omega_{\alpha}\right)-D_{2 j}\left(v^{\prime}, \omega_{\alpha}\right)\right] \mathrm{d} v^{\prime},
$$

$$
0=-\frac{E_{r}}{2 t_{c}}-(\omega-\Omega) E_{i}+\frac{\omega_{0} l}{\epsilon_{1} L} \int_{0}^{\infty} \int_{-\infty}^{\infty} P_{i, 1 i}\left(v, \omega_{\alpha}\right) \mathrm{d} v \mathrm{~d} \omega_{\alpha},
$$




$$
0=-\frac{E_{i}}{2 t_{c}}+(\omega-\Omega) E_{r}-\frac{\omega_{0} l}{\epsilon_{1} L} \int_{0}^{\infty} \int_{-\infty}^{\infty} P_{r, 1 i}\left(v, \omega_{\alpha}\right) \mathrm{d} v \mathrm{~d} \omega_{\alpha},
$$

where Eqs. (29) and (30) were used in reducing the polarization terms in the field equations.

Equations (43) and (44) can be written more compactly in the form

$$
\begin{aligned}
P_{i, 2 j+1}\left(v, \omega_{\alpha}\right)= & -x_{j} P_{r, 2 j+1}\left(v, \omega_{\alpha}\right) \\
& -i \frac{\mu^{2} E_{i}}{2 \hbar\left(\omega-\omega_{\alpha}\right)}\left[D_{2 j}\left(v, \omega_{\alpha}\right)-D_{2 j+2}\left(v, \omega_{\alpha}\right)\right],
\end{aligned}
$$

$$
\begin{aligned}
P_{r, 2 j+1}\left(v, \omega_{\alpha}\right)= & x_{j} P_{i, 2 j+1}\left(v, \omega_{\alpha}\right) \\
& -\frac{i \mu^{2} E_{r}}{2 \hbar\left(\omega-\omega_{\alpha}\right)}\left[D_{2 j}\left(v, \omega_{\alpha}\right)-D_{2 j+2}\left(v, \omega_{\alpha}\right)\right],
\end{aligned}
$$

where $x_{j}$ is the dimensionless parameter

$$
x_{j}=\frac{(2 j+1) i k v+\gamma}{\omega-\omega_{\alpha}} .
$$

Equations (49) and (50) may now be combined to obtain the polarization components

$$
\begin{aligned}
P_{i, 2 j+1}\left(v, \omega_{\alpha}\right)=\frac{i \mu^{2}}{2 \hbar\left(\omega-\omega_{\alpha}\right)\left(1+x_{j}^{2}\right)}\left(E_{r} x_{j}-E_{i}\right) \\
\quad \times\left[D_{2 j}\left(v, \omega_{\alpha}\right)-D_{2 j+2}\left(v, \omega_{\alpha}\right)\right], \\
P_{r, 2 j+1}\left(v, \omega_{\alpha}\right)=\frac{i \mu^{2}}{2 \hbar\left(\omega-\omega_{\alpha}\right)\left(1+x_{j}^{2}\right)}\left(-E_{i} x_{j}-E_{r}\right) \\
\quad \times\left[D_{2 j}\left(v, \omega_{\alpha}\right)-D_{2 j+2}\left(v, \omega_{\alpha}\right)\right] .
\end{aligned}
$$

When Eqs. (52) and (53) are substituted into Eq. (45) we obtain

$$
\begin{aligned}
& 0=\left[\lambda_{a}\left(v, \omega_{\alpha}\right)-\lambda_{b}\left(v, \omega_{\alpha}\right)\right] \delta_{j 0}-\left[(2 j) i k v+h_{1}\right] D_{2 j}\left(v, \omega_{\alpha}\right) \\
& -h_{2} M_{2 j}\left(v, \omega_{\alpha}\right)+\frac{\mu^{2}\left(E_{r}^{2}+E_{i}^{2}\right)}{4 \hbar^{2}\left(\omega-\omega_{\alpha}\right)} \\
& \times\left\{\frac{x_{j-1}}{1+x_{j-1}{ }^{2}}\left[D_{2 j-2}\left(v, \omega_{\alpha}\right)-D_{2 j}\left(v, \omega_{\alpha}\right)\right]\right. \\
& \left.-\frac{x_{j}}{1+x_{j}^{2}}\left[D_{2 j}\left(v, \omega_{\alpha}\right)-D_{2 j+2}\left(v, \omega_{\alpha}\right)\right]\right\} \\
& +\frac{\Gamma_{a}}{2 u \pi^{1 / 2}} \exp \left(-v^{2} / u^{2}\right) \int_{-\infty}^{\infty}\left[M_{2 j}\left(v^{\prime}, \omega_{\alpha}\right)+D_{2 j}\left(v^{\prime}, \omega_{\alpha}\right)\right] \mathrm{d} v^{\prime} \\
& -\frac{\Gamma_{b}}{2 u \pi^{1 / 2}} \exp \left(-v^{2} / u^{2}\right) \int_{-\infty}^{\infty}\left[M_{2 j}\left(v^{\prime}, \omega_{\alpha}\right)-D_{2 j}\left(v^{\prime}, \omega_{\alpha}\right)\right] \mathrm{d} v^{\prime} .
\end{aligned}
$$

For compactness, it is helpful to define the new pump rates

$$
\begin{array}{r}
\lambda_{a, j}\left(v, \omega_{\alpha}\right)=\left\{L_{a}\left(\omega_{\alpha}\right) \delta_{j 0}+\frac{\Gamma_{a}}{2} \int_{-\infty}^{\infty}\left[M_{2 j}\left(v^{\prime}, \omega_{\alpha}\right)+D_{2 j}\left(v, \omega_{\alpha}\right)\right] \mathrm{d} v^{\prime}\right\} \\
\times \frac{\exp \left(-v^{2} / u^{2}\right)}{u \pi^{1 / 2}},
\end{array}
$$

$$
\begin{array}{r}
\lambda_{b, j}^{\prime}\left(v, \omega_{\alpha}\right)=\left\{L_{b}\left(\omega_{\alpha}\right) \delta_{j 0}+\frac{\Gamma_{b}}{2} \int_{-\infty}^{\infty}\left[M_{2 j}\left(v^{\prime}, \omega_{\alpha}\right)-D_{2 j}\left(v^{\prime}, \omega_{\alpha}\right)\right] \mathrm{d} v^{\prime}\right\} \\
\times \frac{\exp \left(-v^{2} / u^{2}\right)}{u \pi^{1 / 2}},
\end{array}
$$

where the Gaussian velocity distribution in the pump functions has been factored out according to

$$
\begin{aligned}
& \lambda_{a}\left(v, \omega_{\alpha}\right)=L_{a}\left(\omega_{\alpha}\right) \exp \left(-v^{2} / u^{2}\right) / u \pi^{1 / 2}, \\
& \lambda_{b}\left(v, \omega_{\alpha}\right)=L_{b}\left(\omega_{\alpha}\right) \exp \left(-v^{2} / u^{2}\right) / u \pi^{1 / 2} .
\end{aligned}
$$

With this notation, Eqs. (54) and (46) simplify to

$$
\begin{aligned}
0= & \lambda_{a, j}{ }^{\prime}\left(v, \omega_{\alpha}\right)-\lambda_{b, j}{ }^{\prime}\left(v, \omega_{\alpha}\right) \\
& -\left[(2 j) i k v+h_{1}\right] D_{2 j}\left(v, \omega_{\alpha}\right)-h_{2} M_{2 j}\left(v, \omega_{\alpha}\right) \\
& +\frac{\mu^{2}\left(E_{r}^{2}+E_{i}^{2}\right)}{4 \hbar^{2}\left(\omega-\omega_{\alpha}\right)}\left\{\frac{x_{j-1}}{1+x_{j-1}^{2}}\left[D_{2 j-2}\left(v, \omega_{\alpha}\right)-D_{2 j}\left(v, \omega_{\alpha}\right)\right]\right. \\
& \left.-\frac{x_{j}}{1+x_{j}^{2}}\left[D_{2 j}\left(v, \omega_{\alpha}\right)-D_{2 j+2}\left(v, \omega_{\alpha}\right)\right]\right\} \\
0= & \lambda_{a, j}{ }^{\prime}\left(v, \omega_{\alpha}\right)+\lambda_{b, j}{ }^{\prime}\left(v, \omega_{\alpha}\right)-\left[(2 j) i k v+h_{3}\right] M_{2 j}\left(v, \omega_{\alpha}\right) \\
& \quad-h_{4} D_{2 j}\left(v, \omega_{\alpha}\right) .
\end{aligned}
$$
is

Equation (60) may be solved for $M_{2 j}\left(v, \omega_{\alpha}\right)$, and the result

$$
M_{2 j}\left(v, \omega_{\alpha}\right)=\frac{\lambda_{a, j}{ }^{\prime}\left(v, \omega_{\alpha}\right)+\lambda_{b, j}{ }^{\prime}\left(v, \omega_{\alpha}\right)-h_{4} D_{2 j}\left(v, \omega_{\alpha}\right)}{(2 j) i k v+h_{3}} .
$$

When Eq. (61) is substituted into Eq. (59), we obtain the expression

$$
\begin{aligned}
D_{2 j}\left(v, \omega_{\alpha}\right)= & \left\{\left[(2 j) i k v+h_{3}-h_{2}\right] \lambda_{a, j}{ }^{\prime}\left(v, \omega_{\alpha}\right)\right. \\
& \left.-\left[(2 j) i k v+h_{3}+h_{2}\right] \lambda_{b, j}{ }^{\prime}\left(v, \omega_{\alpha}\right)\right\} \\
& \div\left\{\left[(2 j) i k v+h_{1}\right]\left[(2 j) i k v+h_{3}\right]-h_{2} h_{4}\right\} \\
& +\left[\frac{\mu^{2}}{4 \hbar^{2}\left(\omega-\omega_{\alpha}\right)}\right] \\
& \times \frac{\left[(2 j) i k v+h_{3}\right]\left(E_{r}{ }^{2}+E_{i}{ }^{2}\right)}{\left[(2 j) i k v+h_{1}\right]\left[(2 j) i k v+h_{3}\right]-h_{2} h_{4}} \\
& \times\left\{\frac{x_{j-1}}{1+x_{j-1}{ }^{2}}\left[D_{2 j-2}\left(v, \omega_{\alpha}\right)-D_{2 j}\left(v, \omega_{\alpha}\right)\right]\right. \\
& \left.-\frac{x_{j}}{1+x_{j}{ }^{2}}\left[D_{2 j}\left(v, \omega_{\alpha}\right)-D_{2 j+2}\left(v, \omega_{\alpha}\right)\right]\right\},
\end{aligned}
$$

Except for the cross-relaxation terms that are implicit in the primed pump rates, the preceding equations can be used to develop a continued fraction expression for the population difference. However, for the purpose of obtaining an effective normalization, it is necessary to consider only the equation for the $j=0$ harmonic of the population difference. The fundamental spatial component of Eq. (62) is 


$$
\begin{aligned}
D_{0}\left(v, \omega_{\alpha}\right)= & \frac{\left(h_{3}-h_{2}\right) \lambda_{a, 0}{ }^{\prime}\left(v, \omega_{\alpha}\right)-\left(h_{3}+h_{2}\right) \lambda_{b, 0}{ }^{\prime}\left(v, \omega_{\alpha}\right)}{h_{1} h_{3}-h_{2} h_{4}} \\
& +\left[\frac{\mu^{2}}{4 \hbar^{2}\left(\omega-\omega_{\alpha}\right)}\right] \frac{h_{3}\left(E_{r}{ }^{2}+E_{i}{ }^{2}\right)}{h_{1} h_{3}-h_{2} h_{4}} \\
& \times\left\{\frac{x_{-1}}{1+x_{-1}{ }^{2}}\left[D_{-2}\left(v, \omega_{\alpha}\right)-D_{0}\left(v, \omega_{\alpha}\right)\right]\right. \\
& \left.-\frac{x_{0}}{1+x_{0}{ }^{2}}\left[D_{0}\left(v, \omega_{\alpha}\right)-D_{2}\left(v, \omega_{\alpha}\right)\right]\right\} .
\end{aligned}
$$

When the definition of the $x$ parameters, as given in Eq. (51), is used, the coefficients of $D_{0}$ in Eq. (63) can be grouped together to yield

$\left\{1+\frac{\mu^{2}}{4 \hbar^{2}} \frac{h_{3}\left(E_{r}^{2}+E_{i}^{2}\right)}{\left(h_{1} h_{3}-h_{2} h_{4}\right) \gamma}\left[\frac{1-i k v / \gamma}{\left(\omega-\omega_{\alpha}\right)^{2} / \gamma^{2}+(1-i k v / \gamma)^{2}}\right.\right.$

$$
\begin{aligned}
& \left.\left.+\frac{1+i k v / \gamma}{\left(\omega-\omega_{\alpha}\right)^{2} / \gamma^{2}+(1+i k v / \gamma)^{2}}\right]\right\} D_{0}\left(v, \omega_{\alpha}\right) \\
& =\frac{\left(h_{3}-h_{2}\right) \lambda_{a, 0}{ }^{\prime}\left(v, \omega_{\alpha}\right)-\left(h_{3}+h_{2}\right) \lambda_{b, 0}{ }^{\prime}\left(v, \omega_{\alpha}\right)}{h_{1} h_{3}-h_{2} h_{4}} \\
& +\frac{\mu^{2}}{4 \hbar^{2}} \frac{h_{3}\left(E_{r}^{2}+E_{i}^{2}\right)}{\left(h_{1} h_{3}-h_{2} h_{4}\right) \gamma} \\
& \times\left[\frac{1-i k v / \gamma}{\left(\omega-\omega_{\alpha}\right)^{2} / \gamma^{2}+(1-i k v / \gamma)^{2}} D_{-2}\left(v, \omega_{\alpha}\right)\right. \\
& \left.+\frac{1+i k v / \gamma}{\left(\omega-\omega_{\alpha}\right)^{2} / \gamma^{2}+(1+i k v / \gamma)^{2}} D_{2}\left(v, \omega_{\alpha}\right)\right] .
\end{aligned}
$$

The form of Eq. (64) suggests introducing the normalized field components

$$
\begin{aligned}
A_{r} & =\frac{\mu}{2 \hbar}\left[\frac{h_{3}}{\left(h_{1} h_{3}-h_{2} h_{4}\right) \gamma}\right]^{1 / 2} E_{r} \\
& =\frac{\mu}{2 \hbar}\left[\frac{\gamma_{a}^{\prime}-\gamma_{a b}+\gamma_{b}^{\prime}}{2 \gamma \gamma_{a}^{\prime} \gamma_{b}^{\prime}}\right]^{1 / 2} E_{r}, \\
A_{i} & =\frac{\mu}{2 \hbar}\left[\frac{h_{3}}{\left(h_{1} h_{3}-h_{2} h_{4}\right) \gamma}\right]^{1 / 2} E_{i} \\
& =\frac{\mu}{2 \hbar}\left[\frac{\gamma_{a}^{\prime}-\gamma_{a b}+\gamma_{b}^{\prime}}{2 \gamma \gamma_{a}^{\prime} \gamma_{b}^{\prime}}\right]^{1 / 2} E_{i} .
\end{aligned}
$$

For a laser with negligible Doppler broadening $(k v \ll \gamma)$, a single atomic frequency class $\left(\omega_{\alpha}=\omega_{0}\right)$, and line-center tuning $\left(\omega=\omega_{0}\right)$, Eq. (64) would now reduce to

$$
\begin{aligned}
&\left\{1+2\left(A_{r}{ }^{2}+A_{i}{ }^{2}\right)\right\} D_{0}\left(v, \omega_{\alpha}\right) \\
&= \frac{\left(h_{3}-h_{2}\right) \lambda_{a, 0}{ }^{\prime}\left(v, \omega_{\alpha}\right)-\left(h_{3}+h_{2}\right) \lambda_{b, 0}\left(v, \omega_{\alpha}\right)}{h_{1} h_{3}-h_{2} h_{4}} \\
&+\left(A_{r}{ }^{2}+A_{i}{ }^{2}\right)\left[D_{-2}\left(v, \omega_{\alpha}\right)+D_{2}\left(v, \omega_{\alpha}\right)\right] .
\end{aligned}
$$

Thus, with this normalization of the field amplitudes, the fundamental harmonic of the population difference seems (at least superficially) to be saturated by twice the normalized intensity $I=A^{2}=A_{r}{ }^{2}+A_{i}{ }^{2}$. The factor of 2 can be associated with the existence of right- and left-travelingwave components, and in the absence of spectral cross relaxation and direct interlevel decay this field normalization reduces to the one that we used previously. ${ }^{22,23}$

It is now possible to write the dynamic equations of the laser in terms of the normalized field amplitudes that have been defined in Eqs. (65) and (66). It is also helpful to introduce normalizations of the other parameters that will permit the most compact expression of the laser equations. In this way we are led to the new normalizations for the polarization, population, and pump variables:

$$
\begin{aligned}
& P_{i, j}(V, U)=\frac{\epsilon u \gamma t_{c} \omega_{0} l \mu}{\epsilon_{1} L \hbar}\left(\frac{\gamma_{a}^{\prime}-\gamma_{a b}+\gamma_{b}^{\prime}}{2 \gamma \gamma_{a}^{\prime} \gamma_{b}^{\prime}}\right)^{1 / 2} P_{i, j}\left(v, \omega_{\alpha}\right), \\
& P_{r, j}(V, U)=\frac{\epsilon u \gamma t_{c} \omega_{0} l \mu}{\epsilon_{1} L \hbar}\left(\frac{\gamma_{a}^{\prime}-\gamma_{a b}+\gamma_{b}^{\prime}}{2 \gamma \gamma_{a}^{\prime} \gamma_{b}^{\prime}}\right)^{1 / 2} P_{r, j}\left(v, \omega_{\alpha}\right), \\
& D_{2 j}(V, U)=\frac{\epsilon u t_{c} \omega_{0} l \mu^{2}}{\epsilon_{1} L \hbar} D_{2 j}\left(v, \omega_{\alpha}\right) \\
& M_{2 j}(V, U)=\frac{\epsilon u t_{c} \omega_{0} l \mu^{2}}{\epsilon_{1} L \hbar} M_{2 j}\left(v, \omega_{\alpha}\right) \\
& \lambda_{a}(V, U)=\frac{\epsilon u t_{c} \omega_{0} l \mu^{2}}{\epsilon_{1} L \hbar} \lambda_{a}\left(v, \omega_{\alpha}\right) \\
& \lambda_{b}(V, U)=\frac{\epsilon u t_{c} \omega_{0} l \mu^{2}}{\epsilon_{1} L \hbar} \lambda_{b}\left(v, \omega_{\alpha}\right)
\end{aligned}
$$

where we have introduced the new variables

$$
\begin{aligned}
& V=\frac{v}{\epsilon u}=\frac{k v}{\gamma}, \\
& U=\frac{\omega_{\alpha}-\omega_{0}}{\gamma},
\end{aligned}
$$

and $\epsilon=\left(\Delta \nu_{h} / \Delta \nu_{d}\right)(\ln 2)^{1 / 2}$ is the natural damping ratio. It is also helpful to introduce the normalized lasing frequency $y$ $=\left(\omega-\omega_{0}\right) / \gamma$, the normalized cavity frequency $y_{0}=(\Omega-$ $\left.\omega_{0}\right) \gamma$, the new decay rate $\gamma_{1}=2 \gamma_{a}^{\prime} \gamma_{b}^{\prime} /\left(\gamma_{a}^{\prime}-\gamma_{a b}+\gamma_{b}{ }^{\prime}\right)$, and the decay parameter $\delta=2 \gamma t_{c}$. With this notation Eqs. (33)(38) take on the relatively simple forms

$$
\begin{aligned}
\frac{\partial P_{r, 2 j+1}(V, U, t)}{\partial t}= & -\gamma\left\{[1+(2 j+1) i V] P_{r, 2 j+1}(V, U, t)\right. \\
& +(y-U) P_{i, 2 j+1}(V, U, t) \\
& \left.+i A_{i}(t)\left[D_{2 j}(V, U, t)-D_{2 j+2}(V, U, t)\right]\right\},
\end{aligned}
$$




$$
\begin{aligned}
\frac{\partial P_{i, 2 j+1}(V, U, t)}{\partial t}= & -\gamma\left\{[1+(2 j+1) i V] P_{i, 2 j+1}(V, U, t)\right. \\
& -(y-U) P_{r, 2 j+1}(V, U, t) \\
& \left.-i A_{r}(t)\left[D_{2 j}(V, U, t)-D_{2 j+2}(V, U, t)\right]\right\},
\end{aligned}
$$

$$
\begin{gathered}
P_{i, 2 j+1}(V, t)=\int_{-\infty}^{\infty} P_{i, 2 j+1}(V, U, t) \mathrm{d} U, \\
D_{2 j}(V, t)=\int_{-\infty}^{\infty} D_{2 j}(V, U, t) \mathrm{d} U,
\end{gathered}
$$

$$
\begin{aligned}
& \frac{\partial D_{2 j}(V, U, t)}{\partial t}=\left[\lambda_{a}(V, U, t)-\lambda_{b}(V, U, t)\right] \delta_{j 0} \\
& \quad-\left[h_{1}+(2 j) i \gamma V\right] D_{2 j}(V, U, t)-h_{2} M_{2 j}(V, U, t) \\
& \quad-i \gamma_{1}\left\{\left[A_{r}(t) P_{i, 2 j-1}(V, U, t)-A_{i}(t) P_{r, 2 j-1}(V, U, t)\right]-\left[A_{r}(t) P_{i, 2 j+1}(V, U, t)-A_{i}(t) P_{r, 2 j+1}(V, U, t)\right]\right\} \\
& \quad+\frac{\epsilon \Gamma_{a}}{2 \pi^{1 / 2}} \exp \left(-\epsilon^{2} V^{2}\right) \int_{-\infty}^{\infty}\left[M_{2 j}\left(V^{\prime}, U, t\right)+D_{2 j}\left(V^{\prime}, U, t\right)\right] \mathrm{d} V^{\prime}-\frac{\epsilon \Gamma_{b}}{2 \pi^{1 / 2}} \exp \left(-\epsilon^{2} V^{2}\right) \int_{-\infty}^{\infty}\left[M_{2 j}\left(V^{\prime}, U, t\right)-D_{2 j}\left(V^{\prime}, U, t\right)\right] \mathrm{d} V^{\prime},
\end{aligned}
$$

$\frac{\partial M_{2 j}(V, U, t)}{\partial t}=\left[\lambda_{a}(V, U, t)+\lambda_{b}(V, U, t)\right] \delta_{j 0}-\left[h_{3}+(2 j) i \gamma V\right] M_{2 j}(V, U, t)-h_{4} D_{2 j}(V, U, t)$

$$
+\frac{\epsilon \Gamma_{a}}{2 \pi^{1 / 2}} \exp \left(-\epsilon^{2} V^{2}\right) \int_{-\infty}^{\infty}\left[M_{2 j}\left(V^{\prime}, U, t\right)+D_{2 j}\left(V^{\prime}, U, t\right)\right] \mathrm{d} V^{\prime}+\frac{\epsilon \Gamma_{b}}{2 \pi^{1 / 2}} \exp \left(-\epsilon^{2} V^{2}\right) \int_{-\infty}^{\infty}\left[M_{2 j}\left(V^{\prime}, U, t\right)-D_{2 j}\left(V^{\prime}, U, t\right)\right] \mathrm{d} V^{\prime}
$$

$$
\begin{aligned}
\frac{\mathrm{d} A_{r}(t)}{\mathrm{d} t}=-\frac{1}{2 t_{c}} & {\left[A_{r}(t)+\delta\left(y-y_{0}\right) A_{i}(t)\right.} \\
& \left.-\int_{-\infty}^{\infty} \int_{-\infty}^{\infty} P_{i, 1 i}(V, U, t) \mathrm{d} V \mathrm{~d} U\right],
\end{aligned}
$$

$$
\begin{gathered}
M_{2 j}(V, t)=\int_{-\infty}^{\infty} M_{2 j}(V, U, t) \mathrm{d} U, \\
\lambda_{a}(V, t)=\int_{-\infty}^{\infty} \lambda_{a}(V, U, t) \mathrm{d} U, \\
\lambda_{b}(V, t)=\int_{-\infty}^{\infty} \lambda_{b}(V, U, t) \mathrm{d} U .
\end{gathered}
$$

$$
\begin{aligned}
\frac{\mathrm{d} A_{i}(t)}{\mathrm{d} t}=-\frac{1}{2 t_{c}}\left[A_{i}(t)-\right. & \delta\left(y-y_{0}\right) A_{r}(t) \\
& \left.+\int_{-\infty}^{\infty} \int_{-\infty}^{\infty} P_{r, 1 i}(V, U, t) \mathrm{d} V \mathrm{~d} U\right] .
\end{aligned}
$$

Because the normalized atomic center frequency $U$ can be negative, the lower limit on the integration over $U$ has been extended to minus infinity.

Equations (76)-(81) allow for the possibility of a distribution of the natural transition center frequencies ( $U$ in the normalized units). However, our initial interest is in the pulsation data for monoisotopic xenon lasers. Consequently the polarization and population variables may be regarded here as delta functions of $U$ and can be replaced with the new variables

$$
P_{r, 2 j+1}(V, t)=\int_{-\infty}^{\infty} P_{r, 2 j+1}(V, U, t) \mathrm{d} U,
$$

With these variable changes and with $U$ set equal to zero, Eqs. (76)-(81) reduce to

$$
\begin{aligned}
\frac{\partial P_{r, 2 j+1}(V, t)}{\partial t}= & -\gamma\left\{[1+(2 j+1) i V] P_{r, 2 j+1}(V, t)\right. \\
& +y P_{i, 2 j+1}(V, t) \\
& \left.+i A_{i}(t)\left[D_{2 j}(V, t)-D_{2 j+2}(V, t)\right]\right\},
\end{aligned}
$$

$$
\begin{aligned}
\frac{\partial P_{i, 2 j+1}(V, t)}{\partial t}= & -\gamma\left\{[1+(2 j+1) i V] P_{i, 2 j+1}(V, t)\right. \\
& -y P_{r, 2 j+1}(V, t) \\
& \left.-i A_{r}(t)\left[D_{2 j}(V, t)-D_{2 j+2}(V, t)\right]\right\},
\end{aligned}
$$

$$
\begin{aligned}
\frac{\partial D_{2 j}(V, t)}{\partial t}= & {\left[\lambda_{a}(V, t)-\lambda_{b}(V, t)\right] \delta_{j 0}-\left[h_{1}+(2 j) i \gamma V\right] D_{2 j}(V, t)-h_{2} M_{2 j}(V, t) } \\
& -i \gamma_{1}\left\{\left[A_{r}(t) P_{i, 2 j-1}(V, t)-A_{i}(t) P_{r, 2 j-1}(V, t)\right]-\left[A_{r}(t) P_{i, 2 j+1}(V, t)-A_{i}(t) P_{r, 2 j+1}(V, t)\right]\right\} \\
& +\frac{\epsilon \Gamma_{a}}{2 \pi^{1 / 2}} \exp \left(-\epsilon^{2} V^{2}\right) \int_{-\infty}^{\infty}\left[M_{2 j}\left(V^{\prime}, t\right)+D_{2 j}\left(V^{\prime}, t\right)\right] \mathrm{d} V^{\prime}-\frac{\epsilon \Gamma_{b}}{2 \pi^{1 / 2}} \exp \left(-\epsilon^{2} V^{2}\right) \int_{-\infty}^{\infty}\left[M_{2 j}\left(V^{\prime}, t\right)-D_{2 j}\left(V^{\prime}, t\right)\right] \mathrm{d} V^{\prime}, \\
\frac{\partial M_{2 j}(V, t)}{\partial t}= & {\left[\lambda_{a}(V, t)+\gamma_{b}(V, t)\right] \delta_{j 0}-\left[h_{3}+(2 j) i \gamma V\right] M_{2 j}(V, t)-h_{4} D_{2 j}(V, t) } \\
& +\frac{\epsilon \Gamma_{a}}{2 \pi^{1 / 2}} \exp \left(-\epsilon^{2} V^{2}\right) \int_{\dot{-}_{\infty}}^{\infty}\left[M_{2 j}\left(V^{\prime}, t\right)+D_{2 j}\left(V^{\prime}, t\right)\right] \mathrm{d} V^{\prime}+\frac{\epsilon \Gamma_{b}}{2 \pi^{1 / 2}} \exp \left(-\epsilon^{2} V^{2}\right) \int_{-\infty}^{\infty}\left[M_{2 j}\left(V^{\prime}, t\right)-D_{2 j}\left(V^{\prime}, t\right)\right] \mathrm{d} V^{\prime},
\end{aligned}
$$




$$
\begin{aligned}
\frac{\mathrm{d} A_{r}(t)}{\mathrm{d} t} & =-\frac{1}{2 t_{c}}\left[A_{r}(t)+\delta\left(y-y_{0}\right) A_{i}(t)-\int_{-\infty}^{\infty} P_{i, 1 i}(V, t) \mathrm{d} V\right], \\
\frac{\mathrm{d} A_{i}(t)}{\mathrm{d} t} & =-\frac{1}{2 t_{c}}\left[A_{i}(t)-\delta\left(y-y_{0}\right) A_{r}(t)+\int_{-\infty}^{\infty} P_{r, 1 i}(V, t) \mathrm{d} V\right] .
\end{aligned}
$$

This reduced form of the equations is the basis for the numerical solutions described below.

\section{INTENSITY SOLUTIONS}

In the previous sections a theoretical model was developed for interpreting and predicting the spontaneous pulsation characteristics of practical standing-wave gas lasers. To apply these results to any specific laser, it is necessary to obtain values for the numerous parameters that appear in the model. Our emphasis here is on the xenon gas laser operating at $3.51-\mu \mathrm{m}$ wavelength, and an effort was made in our previous ring-laser study to glean from the literature the best values for all the parameters of this laser system. ${ }^{5} \mathrm{We}$ list here the xenon parameters that were found previously. These include the upper-state lifetime of approximately $\tau_{a}$ $=1 / \gamma_{a}=1200 \mathrm{nsec}$, the lower-state lifetime of approximate-
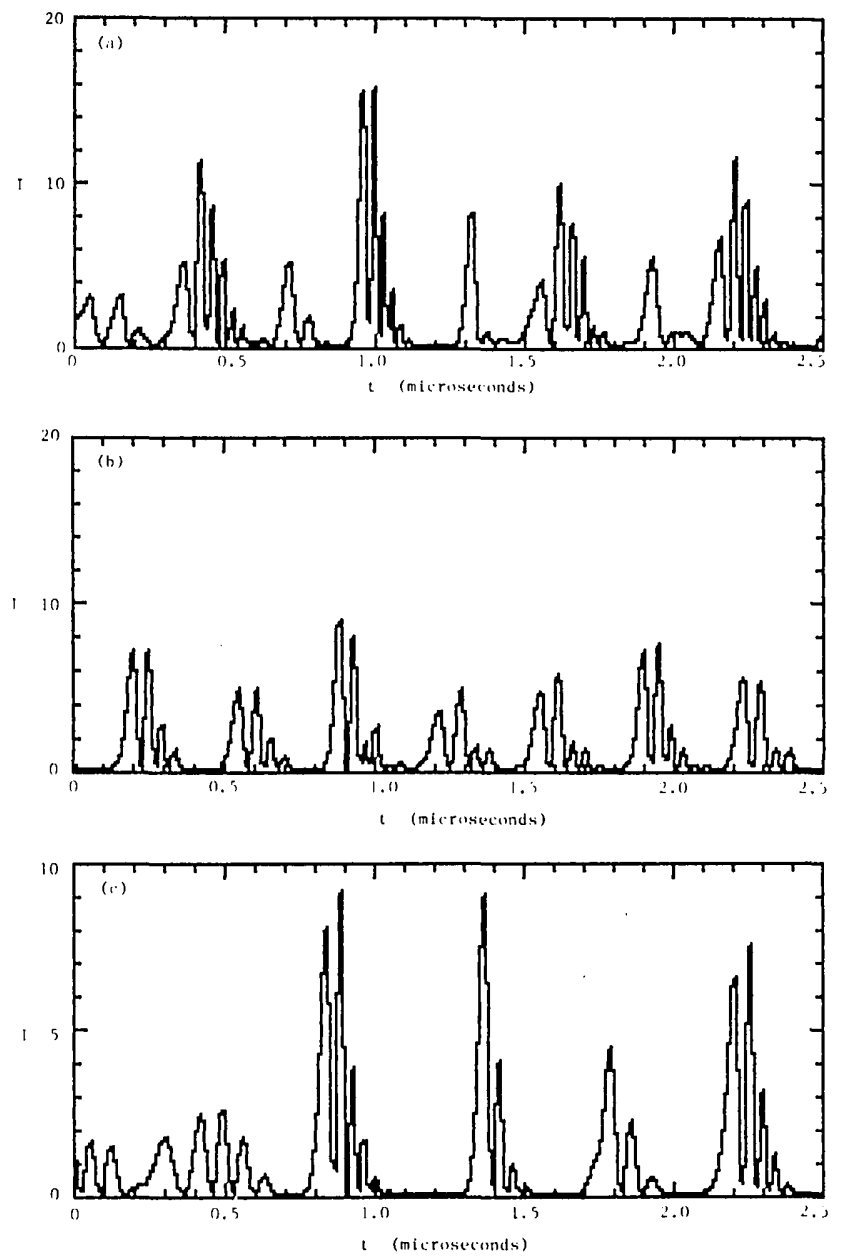

ly $\tau_{b}=1 / \gamma_{b}=35 \mathrm{nsec}$, and the interlevel decay rate $\gamma_{a b}=\gamma_{a}$. Under our operating conditions of approximately 5-mTorr pressure, the phase decay rate is approximately $\gamma=16.3 \times$ $10^{6} \mathrm{sec}^{-1}$, and the spectral cross-relaxation rates are approximately $\Gamma_{a}=\Gamma_{b}=1 \times 10^{6} \mathrm{sec}^{-1}$. At our operating temperature the Doppler width is approximately $\Delta \nu_{d}=110 \mathrm{MHz}$, and the natural damping ratio is approximately $\epsilon \approx 0.039$. The pump-rate ratio is approximately $\lambda_{b} / \lambda_{a}=12.2$, and with our cavity parameters the cavity lifetime is approximately $t_{c}$ $=1.0$ nsec. These are the only parameters needed to carry out a wide range of calculations relating to spontaneous pulsations in low-pressure xenon lasers.

The starting point for our numerical calculations is the equation set of Eqs. (88)-(93). The procedures employed here are similar to those used in our previous studies of Lamb-equation instabilities. ${ }^{4,5,20,22-26}$ In particular, the time integrations were performed by using a modified second-order Runge-Kutta method, and after each time step the velocity integrals were recalculated by using Simpson's rule. Other techniques were also introduced for relating the pump rates to the threshold parameter $r$, for minimizing the computer run time, etc. We intend to report more fully elsewhere on the details of these techniques, the computer requirements, and the various factors that affect the accuracy and relevance of the computed results.
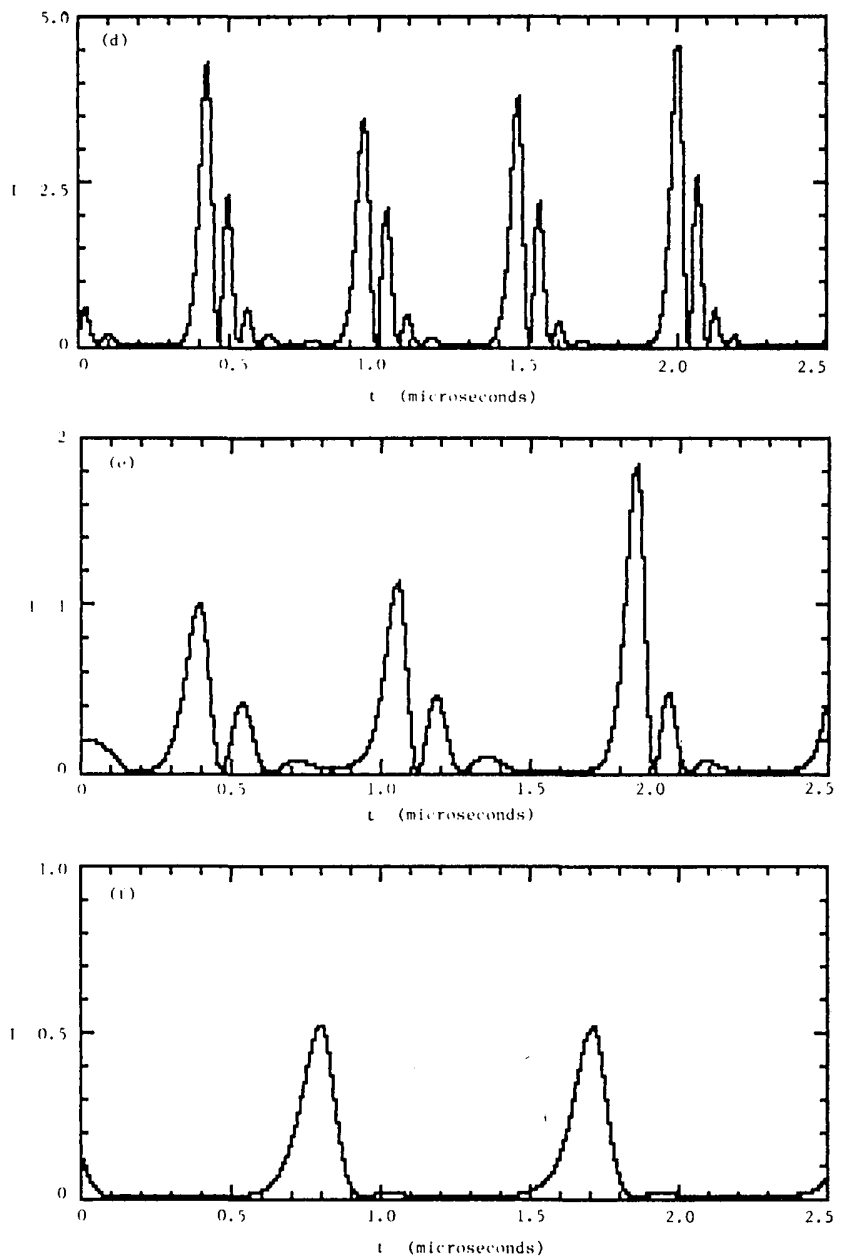

Fig. 1. Theoretical spontaneous pulsation intensity waveforms for a single-mode standing-wave xenon laser with line-center tuning and threshold parameter values of (a) $r=2.0$, (b) $r=1.8$, (c) $r=1.6$, (d) $r=1.4$, (e) $r=1.2$, and (f) $r=1.1$. The amplitude, frequency, and waveform complexity of the pulsations all tend to decrease with decreasing values of $r$. 


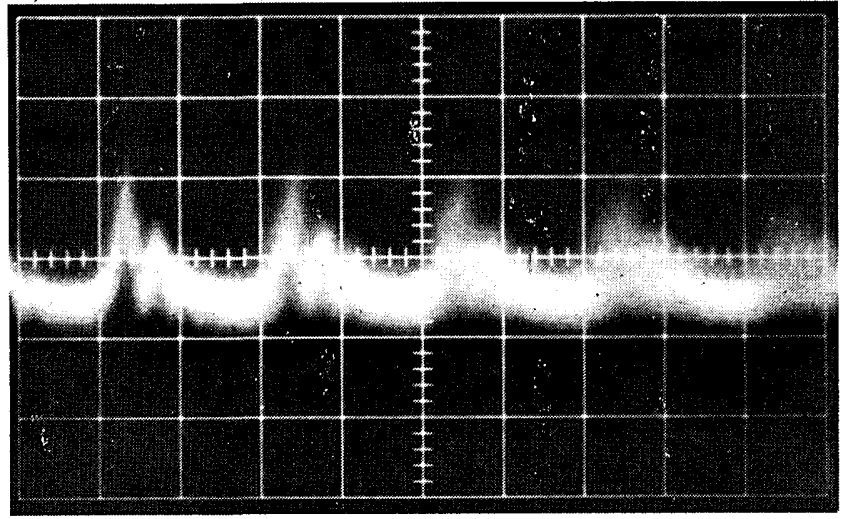

Fig. 2. Experimental time-domain plot of the output intensity from a low-pressure xenon laser at a discharge current of $40 \mathrm{~mA}$ and a time scale of $0.2 \mu \mathrm{sec} /$ division.

The kinds of behavior that might be expected to be observed in a low-pressure standing-wave laser can be best illustrated by initially presenting a series of time-domain intensity waveforms. Such a series is shown in Fig. 1 with line-center tuning and decreasing values of the threshold parameter $r$. In all the present studies the threshold parameter is defined as the ratio of the unsaturated line-center gain to the gain value required to reach threshold for linecenter tuning. The plot of normalized intensity $I$ versus time that is shown in Fig. 1(a) corresponds to a threshold parameter $r=2$ and illustrates many of the possible complexities of xenon-laser behavior. The laser is highly unstable, and the output appears as a semiperiodic train of alternating simple and complicated pulsation bursts. The same kind of pulsation behavior was also observed in numerical solutions of the center-tuned ring lasers, and these results may be compared with the ring-laser results in Refs. 5 and 24. Although the pulsation frequencies are about the same
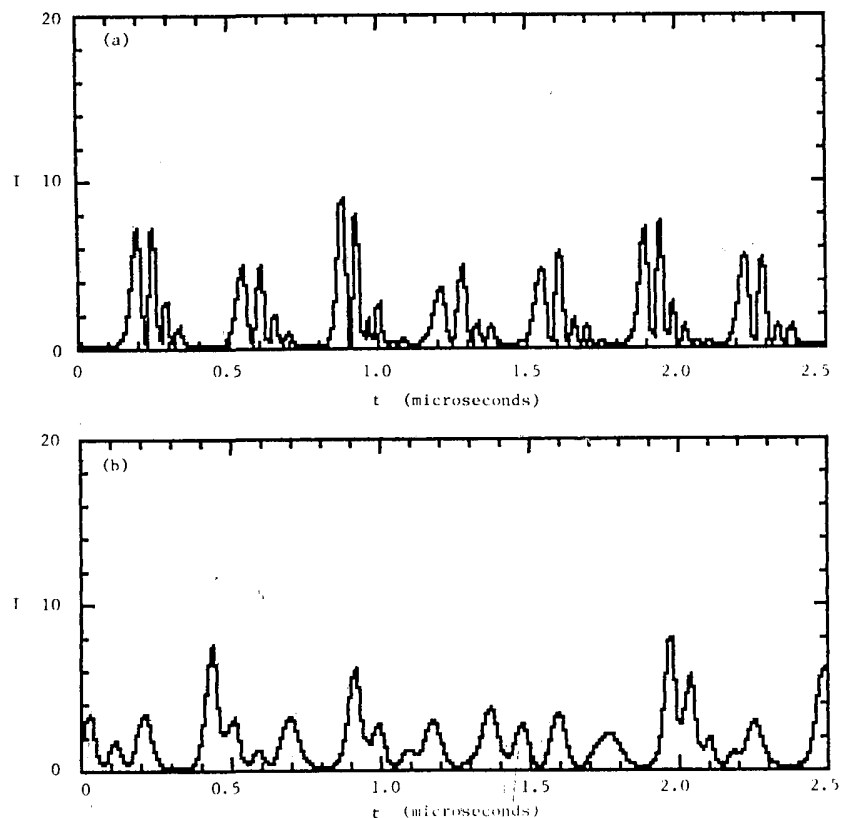

for the standing-wave and ring lasers at $r=2$, the successive pulsation bursts were nearly identical in the ring laser. In other words, the standing-wave laser under these conditions is exhibiting a type of chaotic behavior that would be observed only at significantly higher threshold parameter values $(r \geq 3)$ in an analogous ring laser. For the lower threshold parameter value $r=1.8$, the pulsations shown in Fig. 1(b) do not exhibit the clear alternation between the two types of pulsation bursts that are observed further above threshold, but the intensity is still much less regular than in the corresponding ring laser. The pulsation bursts observed with the smaller threshold parameter values of $r=1.6,1.4,1.2$ are also similar to their ring-laser counterparts, but the standing-wave laser results always show somewhat greater levels of irregularity or chaos. Even with threshold parameter values as low as $r=1.1$ the standing-wave laser is producing discernible pulsation bursts as shown in Fig. 1(f), whereas the corresponding ring laser is reduced to a simple alternating pulse output. In summary then, the center-tuned standing-wave laser produces pulsations of similar frequency to the center-tuned ring laser, but the pulse complexity tends to be of a slightly higher order in the standing-wave laser for any given level of $r$.

It should be noted that the intensity normalization used here is a little different from that used in our ring-laser studies of Refs. 5 and 24. Here we use, as noted above, $I=$ $A_{r}{ }^{2}+A_{i}^{2}$, whereas our ring-laser studies employed

$$
I=\frac{\left(\gamma_{a}-\gamma_{a b}+\gamma_{b}\right) \gamma_{a}{ }^{\prime} \gamma_{b}{ }^{\prime}}{\left(\gamma_{a}^{\prime}-\gamma_{a b}+\gamma_{b}{ }^{\prime}\right) \gamma_{a} \gamma_{b}}\left(A_{r}^{2}+A_{i}^{2}\right)
$$

This previous normalization was chosen for more convenient evaluation of spectral cross-relaxation effects, but we choose here to abandon it. In a quantitative comparison of our standing-wave and ring-laser results with spectral cross relaxation, the ring-laser intensities will thus appear exagger-
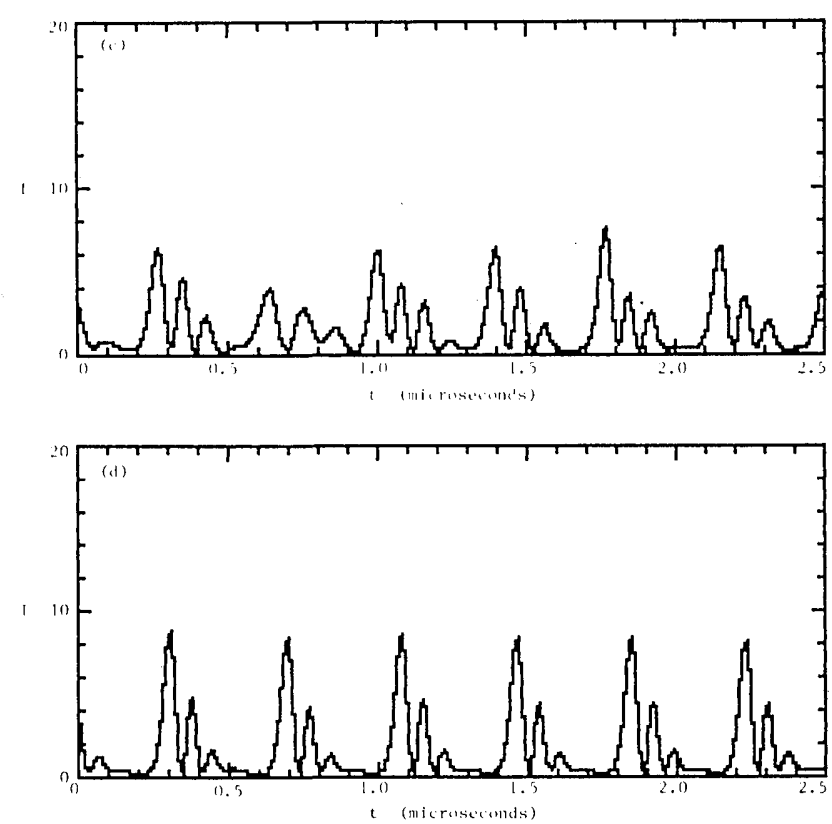

Fig. 3. Theoretical spontaneous pulsation waveforms for a xenon laser with a threshold parameter of $r=1.8$ and detunings of (a) $\Delta \nu=0 \Delta \nu_{h}$, (b) $\Delta \nu=2.5 \Delta \nu_{h}$, (c) $\Delta \nu=5 \Delta \nu_{h}$, and (d) $\Delta \nu=7.5 \Delta \nu_{h}$. In contrast to the ring-laser results, the pulsation intensity and periodicity tend initially to increase with detuning from line center. 


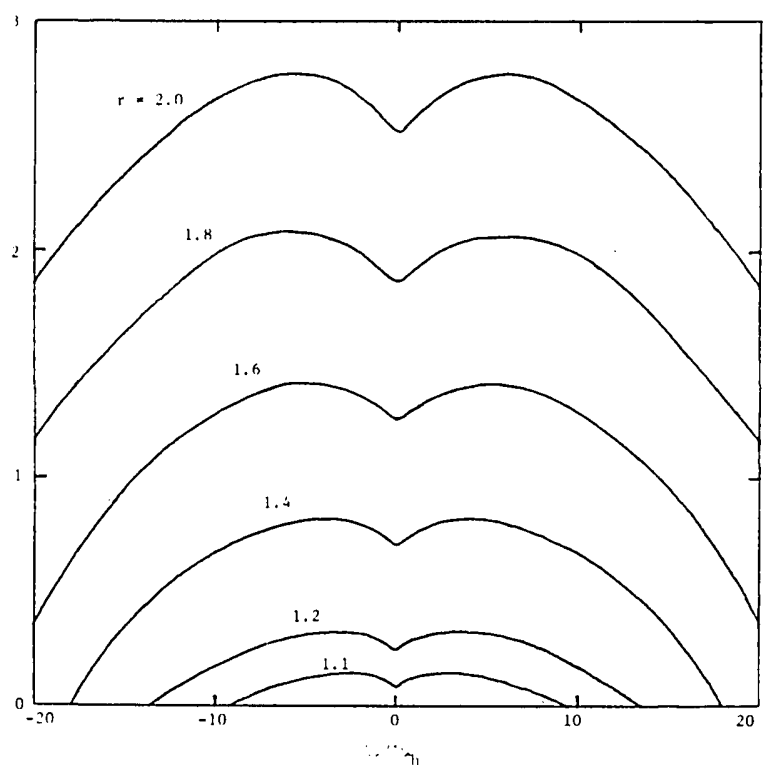

Fig. 4. Theoretical Lamb-dip curves of average intensity versus frequency tuning for a spontaneously pulsing low-pressure xenon laser. The frequency detuning is measured in units of the homogeneous line width $\Delta \nu_{h}$.

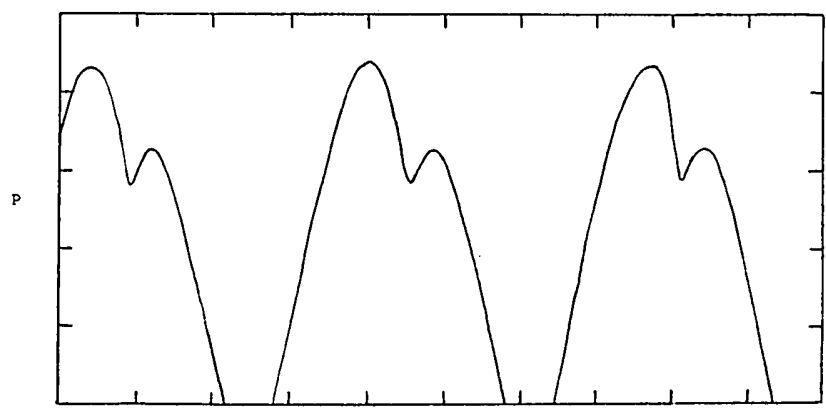

Fig. 5. Experimental Lamb-dip power curve for a low-pressure xenon laser with decreasing cavity length (increasing frequency) and a discharge current of $18 \mathrm{~mA}$ (after Ref. 27).

ated by the factor given in Eq. (94), which, with the present parameter values, evaluates to 2.13 .

The slightly irregular pulsation bursts observed for intermediate threshold parameters in Fig. 1 are also observed in experiments. In Fig. 2 is a multitrace oscilloscope plot of the output intensity from a low-pressure xenon laser. The fuzziness of the trace is due to pulse-to-pulse amplitude variations, as predicted by the theory. The increasing fuzziness to the right shows that the pulsation period is also not quite constant.

The model that was developed here can also describe the effects of cavity detuning on the pulsation characteristics. A series of pulsation waveforms for various values of detuning and a threshold parameter value of $r=1.8$ is shown in Fig. 3. The waveform in Fig. 3(c) corresponds approximately to the experimental conditions yielding the data in Fig. 2. A notable feature of Fig. 3 is that detuning from the Lamb dip at line center tends initially to cause an increase in pulsation-burst periodicity and an increase in average intensity. When we use this model it is straightforward to calcu- late the average intensity as a function of detuning, and a family of Lamb-dip intensity curves is given in Fig. 4 . These tuning curves are similar to experimental Lamb-dip data obtained with xenon lasers except that gain and dispersion focusing effects, neglected in the computations, are sometimes found to cause the Lamb-dip power curves to be asymmetric, as shown in Fig. 5.27 A first-order correction for the asymmetry can be introduced if one multiplies the computed Lamb-dip intensity curves by the square of the frequency-dependent normalized spot size ${ }^{27}$ :

$$
\begin{aligned}
w^{*}(x)=\exp \left(\frac{x^{2}}{4}\right)\left\{\left[1+\left(\frac{2 F(x) \exp x^{2}}{\pi^{1 / 2}}\right)^{2}\right]^{1 / 2}\right. & \\
& \left.+\frac{2 F(x) \exp x^{2}}{\pi^{1 / 2}}\right\}^{-1 / 4},
\end{aligned}
$$

where $F(x)$ has been called Dawson's integral,

$$
F(x)=\exp \left(-x^{2}\right) \int_{0}^{x} \exp \left(t^{2}\right) \mathrm{d} t
$$

and $x=2\left(\nu-\nu_{0}\right)(\ln 2)^{1 / 2} / \Delta \nu_{d}$ is a normalized frequency. With this rescaling the Lamb-dip curves of Fig. 4 are replotted in Fig. 6 and agree well with experimental data. It is significant that the theoretical curves for typical experimental values of the threshold parameter suggest approximately a 10-20\% intensity dip at line center, for this is the same as the experimentally obtained Lamb-dip depth. Only a theoretical model that includes standing-wave fields and spectral cross relaxation can provide a reasonable basis for interpreting such experimental Lamb-dip data.

\section{DISCUSSION}

Within the past few years there has been much progress in the understanding of the instability phenomena that sometimes affect laser oscillators. In spite of that progress, how-

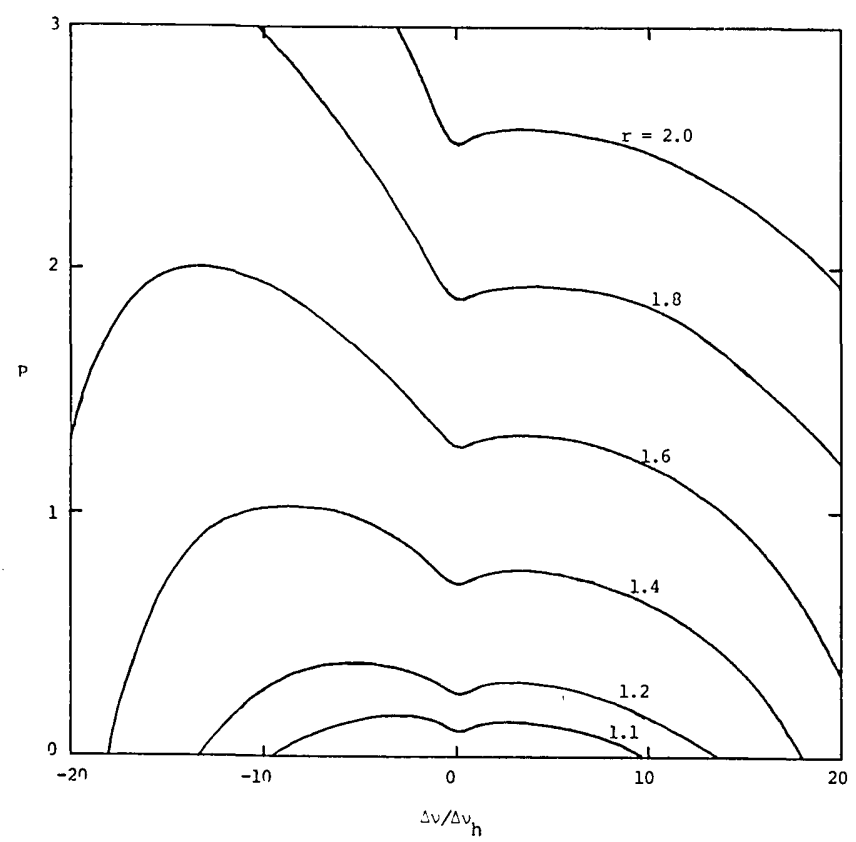

Fig. 6. Theoretical Lamb-dip power curves from Fig. 4 after multiplication by the square of the gain and dispersion focusing correction given in Eq. (95). 
ever, there still remain some significant gaps in the understanding of laser instabilities. One such gap concerns a fundamental assumption that is often made in the development of theoretical instability models. It is usually assumed, for mathematical simplicity, that the electromagnetic fields in the laser are unidirectional traveling waves. On the other hand, most lasers in which instabilities have been observed are better regarded as standing-wave systems, and many of their oscillation characteristics cannot be even qualitatively reproduced by traveling-wave models. The purpose of this study has been to develop a standing-wave model that takes account of the most fundamental physical properties of typical gas-laser systems.

The laser analysis presented here parallels in philosophy our earlier studies of instabilities in ring lasers. Thus it is basically a semiclassical model that includes realistic spontaneous decay and collisional relaxation processes with numerical values appropriate to low-pressure xenon lasers. A particularly significant feature of the model is its ability to yield accurate predictions of the Lamb dip. The implications of the model for the frequency characteristics of the pulsations are reported in another paper in this issue of the journal. ${ }^{21}$

\section{ACKNOWLEDGMENTS}

I am grateful for valuable discussions with N. B. Abraham and D. L. MacFarlane. This research was supported in part by the National Science Foundation and by Tektronix, Inc.

\section{REFERENCES}

1. A. G. Gurtovnik, Izv. Vyssh. Uchebn. Zavedenii Radiofiz. 1, 83 (1958) [English translation TG 230-T382 in Applied Physics Laboratory Library Bulletin (Johns Hopkins University, Baltimore, Md., 1963)].

2. G. Makhov, C. Kikuchi, J. Lambe, and R. W. Terhune, Phys. Rev. 109, 1399 (1958).

3. C. Kikuchi, J. Lambe, G. Makhov, and R. W. Terhune, J. Appl. Phys. 30, 1061 (1959).
4. L. W. Casperson, in Third New Zealand Symposium on Laser Physics, J. D. Harvey and D. F. Walls, eds., Vol. 182 of Springer Lecture Notes in Physics (Springer-Verlag, Berlin, 1983), p. 88.

5. L. W. Casperson, J. Opt. Soc. Am. B 2, 62 (1985).

6. J. C. Englund, R. R. Snapp, and W. C. Schieve, Prog. Opt. 21, 355 (1984).

7. N. B. Abraham, L. A. Lugiato, and L. M. Narducci, J. Opt. Soc. Am. B 2, 7 (1985).

8. J. R. Ackerhalt, P. W. Milonni and M. L. Shih, Phys. Rep. 128, 205 (1985).

9. R. G. Harrison and D. J. Biswas, Prog. Quantum Electron. 10, 147 (1985).

10. N. B. Abraham, P. Mandel, and L. M. Narducci, "Dynamical instabilities and pulsations in lasers," in Progress in Optics, Vol. 25, E. Wolf, ed. (North-Holland, Amsterdam, to be published).

11. J. Bentley and N. B. Abraham, Opt. Commun. 41, 52 (1982).

12. M. Maeda and N. B. Abraham, Phys. Rev. A 26, 3395 (1982).

13. N. B. Abraham, T. Chyba, M. Coleman, R. S. Gioggia, N. J. Halas, L. M. Hoffer, S. N. Liu, M. Maeda, and J. C. Wesson, in Third New Zealand Symposium on Laser Physics, J. D. Harvey and D. F. Walls, eds., Vol. 182 of Springer Lecture Notes in Physics (Springer-Verlag, Berlin, 1983), p. 107.

14. R. S. Gioggia and N. B. Abraham, Phys. Rev. Lett. 51, 650 (1983).

15. R. S. Gioggia and N. B. Abraham, in Coherence and Quantum Optics V, L. Mandel and E. Wolf, eds. (Plenum, New York, 1984), p. 563.

16. L. E. Urbach, S. N. Liu, and N. B. Abraham, in Coherence and Quantum Optics V, L. Mandel and E. Wolf, eds. (Plenum, New York, 1984), p. 593.

17. L. M. Hoffer, T. H. Chyba, and N. B. Abraham, J. Opt. Soc. Am. B 2, 102 (1985).

18. M. F. H. Tarroja, N. B. Abraham, D. K. Bandy, T. Isaacs, R. S. Gioggia, S. P. Adams, L. M. Narducci, and E. A. Lugiato, in Optical Instabilities, R. W. Boyd, M. G. Raymer, and L. M. Narducci, eds. (Cambridge U. Press, Cambridge, 1986), p. 246.

19. M. F. H. Tarroja, N. B. Abraham, D. K. Bandy, and L. M. Narducci, Phys. Rev. A 34, 3148 (1986).

20. L. W. Casperson, IEEE J. Quantum Electron. QE-14, 756 (1978).

21. L. W. Casperson, J. Opt. Soc. Am B 5, 970 (1988):

22. L. W. Casperson, Phys. Rev. A 21, 911 (1980), Eq. (45).

23. L. W. Casperson, Phys. Rev. A 23, 248 (1981), Eq. (87).

24. L. W. Casperson, J. Opt. Soc. Am. B 2, 73 (1985).

25. L. W. Casperson, J. Opt. Soc. Am. B 2, 993 (1985).

26. L. W. Casperson, Opt. Quantúm Electron. 19,'29'(1987).

27. L. W. Casperson and A. Yariv, AppI. Opt. 11, 462 (1972).

\section{Lee W. Casperson}

Lee W. Casperson was born in Portland, Oregon, in 1944. He received the B.S. degree in physics from the Massachusetts Institute of Technology in 1966 and the M.S. and Ph.D. degrees in electrical engineering and physics from the California Institute of Technology in 1967 and 1971, respectively. From 1971 to 1983 he was with the School of Engineering and Applied Science at the University of California at Los Angeles. In 1981 he was a visiting professor in the Department of Physics, University of Auckland, Auckland, New Zealand, and he is now head of the Department of Electrical Engineering, Portland State University, Portland, Oregon. He is an author of more than 100 publications relating primarily to laser physics, light scattering, modulation, and waveguides, and he received the Centennial Medal of the Institute of Electrical and Electronics Engineers for his achievements. As relates to the subject of this special issue, his research includes the experimental discovery and theoretical explanation of the first semiclassical laser instability. 